\title{
MULTI-OBJECTIVE TRANSPORTATION PROBLEM UNDER TYPE-2 TRAPEZOIDAL FUZZY NUMBERS WITH PARAMETERS ESTIMATION AND GOODNESS OF FIT
}

\author{
Murshid KAMAL ${ }^{1}$, Ali ALARJANI ${ }^{2}$, Ahteshamul HAQ ${ }^{3}$, \\ Faiz Noor Khan YUSUFI ${ }^{4}$, Irfan ALI ${ }^{5}$ \\ 1,3,4,5 Dept of Statistics and Operations Research, Aligarh Muslim University, Aligarh, Uttar Pradesh, India \\ ${ }^{2}$ College of Engineering, Prince Sattam Bin Abdulaziz University, Al-Kharj, Saudi Arabia
}

Submitted 31 March 2020; resubmitted 26 June 2020; accepted 27 July 2020

\begin{abstract}
The problem of transportation in real-life is an uncertain multi-objective decision-making problem. In particular, by taking into account the conflicting objectives, Decision-Makers (DMs) are looking for the best transport set up to determine the optimum shipping quantity subject to certain capacity constraints on each route. This paper presented a Multi-Objective Transportation Problem (MOTP) where the objective functions are considered as Type-2 trapezoidal fuzzy numbers (T2TpFN), respectively. Demand and supply in constraints are in multi-choice and probabilistic random variables, respectively. Also considered the "rate of increment in Transportation Cost (TC) and rate of decrement in profit on transporting the products from $i$ th sources to $j$ th destinations due to" (or additional cost) of each product due to the damage, late deliveries, weather conditions, and any other issues. Due to the presence of all these uncertainties, it is not possible to obtain the optimum solution directly, so first, we need to convert all these uncertainties from the model into a crisp equivalent form. The two-phase defuzzification technique is used to transform T2TpFN into a crisp equivalent form. Multi-choice and probabilistic random variables are transformed into an equivalent value using Stochastic Programming (SP) approach and the binary variable, respectively. It is assumed that the supply and demand parameter follows various types of probabilistic distributions like Weibull, Extreme value, Cauchy and Pareto, Normal distribution, respectively. The unknown parameters of probabilistic distributions estimated using the maximum likelihood estimation method at the defined probability level. The best fit of the probability distributions is determined using the Akaike Information Criterion (AIC) and the Bayesian Information Criterion (BIC), respectively. Using the Fuzzy Goal Programming (FGP) method, the final problem is solved for the optimal decision. A case study is intended to provide the effectiveness of the proposed work.
\end{abstract}

Keywords: multi-objective optimization, transportation problem, fuzzy goal programming, multi-choice, maximum likelihood estimation, Akaike information criterion, Bayesian information criterion, stochastic programming.

\section{Notations}

AIC - Akaike information criterion;

ANFIGS - adaptive neuro-fuzzy inference guidance system;

BIC - Bayesian information criterion;

BTP - binary technique procedure;

$\mathrm{CM}$ - congestion management;

DM - decision-maker;

FCP - fuzzy compromise programming;

FGP - fuzzy goal programming;

FLP - fuzzy linear programming;

FMCGP - fuzzy multi-choice goal programming;

FOU - footprint of uncertainty;

\author{
FP - fuzzy programming; \\ FTP - fuzzy transportation problem; \\ GA - genetic algorithm; \\ GP - goal programming; \\ LPP - linear programming problem; \\ MCGP - multi-choice goal programming; \\ MOAP - multi-objective assignment problem; \\ MOTP - multi-objective transportation problem; \\ PDF - probability density function; \\ RHS - right hand side; \\ SP - stochastic programming; \\ T1FS - type-1 fuzzy set;
}

*Corresponding author. E-mail: a.alarjani@psau.edu.sa 
T2FS - type-2 fuzzy set;

T1TpFN - type-1 trapezoidal fuzzy numbers;

T2TpFN - type-2 trapezoidal fuzzy numbers;

TC - transportation cost;

$\mathrm{TP}$ - transport problem;

TPVDS-A - transportation problem with varying demands and supplies-algorithm.

\section{Introduction}

In this, the competitive environment of the business market. Every business is so struggling to discover some lucrative transport strategies to deliver the products to customers.

The TP is a very fascinating management science technique, which can be constructed and solved as a LPP. TP is considered as a logistic or networking problem, with the main purpose is to when and how to deliver products or goods from origins to destinations at the maximum profit and minimum cost.

In many real-life problems, the DM intends to send similar kinds of goods from their origins (also known as plants, sources, supply points) to distinct kinds of destinations (also known as sinks, demand points) to meet the destination demands. In this form of TP, the DMs primary objective is to find the best financial route to transfer a collection of commodities from origin to destination, minimizing the total cost of transportation and transportation time. Dantzig (1963) discussed a TP as linear programming and used simplex method to obtain the optimal solution. Charnes and Cooper (1954) developed a new method known as the stepping stone method and used this method in TP for explaining the linear programming solution procedure for the problem. Appa (1973) expressed many variations of the TP; Arsham and Kahn (1989) considered a general TP and proposed simplex based algorithm for solving TP. Kaliski et al. (1993) developed a short out potential reduction algorithm for solving TP.

In recent years we have been able to recognize that a traditional mathematical programming model is inadequate for many of the real-world circumstances. The nature of these problems demands, on the one hand, that multiple objectives be taken into account. In recent years many researchers have considered multiple objectives in TP such as; Lee and Moore (1973) describe the GP approach to optimize multiple conflicting goals in transportation problems. Aneja and Nair (1979) develop a method of finding the non-dominated extreme points, which involves a parametric search in the criteria space for the bi-criteria transportation problem. Isermann (1979) presented an algorithm, which provides a union of a minimal number of convex sets of all efficient solutions for MOTP. Diaz (1978) described the method for solving the MOTP. Diaz (1979) used a complete description of all efficient solutions to solve the MOTP. Current and Min (1986) included 42 selected references for the bibliography and presented a brief history of the use of multi-objective analysis in transportation planning. Current and Marsh (1993) updated review of multi-objective network design and routing problems that provides the variety of problems addressed reflects the importance and complexity of transportation network analysis. Clímaco et al. (1993) presented TRIMAP method for solving the three-objective transportation problems. TRIMAP seem to be very useful for exploiting an interactive way, a progressive and selective identification of the set of non-dominated solutions. Ringuest and Rinks (1987) presented two interactive algorithms that take advantage of the extraordinary form of the MOTP. Li and Lai (2000) presented a FCP approach to MOTPs at which the synthetic membership degree of the global evaluation for all objectives at maximum. Biswas and De (2018) introduce a process of defuzzification for ranking of T2TpFN in which a two-phase defuzzification method has developed for using PDF of the random variables associates with the fuzzy numbers. FGP technique is used for achieving the highest degree of each of the defined membership goals to the extent possible in the decision-making. Kamal et al. (2018) considered a MOTP and developed a new technique called the distance-based method for solving it.

Inspired by such type of work have been done in multi-criteria decision-making TPs. It is challenging for DMs to make a reasonable assumption about profit and also minimize the total TC if information about the parameters of the real-world problem is precisely not known. In this manuscript, a model is formulated for multi-objective TP in which objective and constraints of the model are uncertain and T2TpNs. We have also considered two cases in constraints of the proposed model: (1) when demand and supply are in interval type multi-choice, and (2) when demand and supply are random variables follows some types of probability distributions. In our proposed model, we have defined a new function in objective functions of the model, the rate of increment in TP cost and rate of decrement in the profit. As per our best knowledge, nobody has discussed these issues in their work.

Other than we have also suggested some proposal in work like AIC and BIC approach to obtain the best fit for probability distributions. The remaining of this paper describe as follows: A brief literature review on TP is defined in Section 1. In Section 2, basic models of TP are discussed. In Section 3, the mathematical formulation MOTP is designed. In Section 4, mixed uncertain constraints of TP and transformation techniques have been used to convert uncertain parameters into certain have been discussed. In Section 5, the stepwise procedure has been discussed for the compromise solution. In Section 6 , the numerical example provided for the justification of proposed work. In Section 7, results and discussion have been discussed. Section 8 provides the managerial implications, contribution and limitations of the proposed work. Finally, conclusions have been discussed for the proposed work. 


\section{Literature review}

In the real-world problems, several diverse situations occur such as uncertain judgments, unpredictable conditions or human error, incomplete knowledge and information and other similar causes, due to which we do not need to get a relevant, precise data about the problem. Such type of impreciseness of any data can be represented in different ways. In such cases, the imprecise data information can be presented in fuzzy numbers. Zadeh (1965) gives the concept of fuzzy set theory. Many researchers: Bit et al. (1992) presented an application of FLP for linear MOTP, and an efficient FORTRAN software (https://fortran-lang.org) has been developed based on the FLP algorithm, which is an extended version of the simplex algorithm. Li and Lai (2000) presented a FCP approach to MOTPs at which the synthetic membership degree of the global evaluation for all objectives at maximum. El-Wahed (2001) described a FP approach to determine the optimal compromise solution of a MOTP, and the fuzzy approach outperforms the interactive procedure as the number of objectives and constraints increases. Dinagar and Palanivel (2009) considered a TP in vagueness and used trapezoidal fuzzy numbers. FTP converted into crisp by Ebrahimnejad (2014) and solved by the standard transportation algorithm for reduction of the computational complexity of the existing method. Gupta and Kumar (2012) proposed a new method for a linear MOTP by shortcomings of the existing method. Zangiabadi and Maleki (2013) used hyperbolic and exponential membership functions to solve the MOTP, which gives an optimal compromise solution. The obtained result has compared with the solution obtained by using a linear membership function.

Rani and Gulati (2014) used unbalanced (availability $<$ demand) fully FTPs and proposed a method for solving unbalanced fully FTPs. Some others researchers use fuzzy set theory in other research areas as Pamucar and Ćirović (2018) considered the vehicle route selection problem under some uncertainties and used ANFIGS technique to determine the optimum route for the transportation. Biswas and Pal (2019) consider CM problem in electric power transmission lines by employing a GA and also used FGP to get an optimum solution. Lukovac and Popović (2018) presented the fuzzy delphi approach to defining a cycle for assessing the performance of military drivers. The approach is modelled to take into account the importance weight of each DM and the homogeneity of the individual fuzzy preferences and used fuzzy triangular numbers. Vilela et al. (2019) described the value of information methodology by integrating fuzzy logic into the decisionmaking process, develop human thinking assessment and coherently combine several economic criteria. Fuzzy inference system in the domain of value of information for methodology is applied to an oil and gas subsurface assessment in which results of the standard and fuzzy methodologies are compared. Si et al. (2019) compared the picture fuzzy numbers using score and accuracy values when the values are equal. The proposed ranking method based on positive ideal solution, positive and negative goal differences, and score and accuracy degrees of the picture fuzzy numbers. A new score function proposed for calculating the actual score value, which depends on the positive and negative goal differences and the neutral degree. Stojić et al. (2018) determined the fuzzy model for the renewal problem of truck road freight vehicle fleet and the assessment of the period needed for a return on the investment. Fazlollahtabar (2018) proposed a substantial operation to fulfil the reverse chain for minimizing the total cost of reverse supply chain and formulated the mathematical model for return product in reverse supply chain considering quality assurance.

In several real-life situations where parameters of decision-making problems have multiple choices. In those situations where DMs are allowed to set a goal of multiple choices. Sometimes, real-world TP has several multiple choices for some parameters. Chang $(2007,2008)$ was first who used the concept of the multi-choice parameter in decision-making problem. Biswal and Acharya (2009) discussed a multi-choice multi-objective LPP in which the RHS parameters of the constraints are multi-choice. The formulated mathematical model solved with the help of existing nonlinear programming method. Biswal and Acharya (2011) described multi-choice programming in which the RHS parameters are multi-choice as functional values of an affine function at some non-negative integer nodes. The interpolating polynomials of all multi-choice parameters formulated as a mathematical programming problem, which is treated as a non-LPP involving mixedinteger type variables. Dutta and Murthy (2010) considered a TP where the objective is expressed in multi-choices and constraints are in fuzzy. They also discussed two cases and proposed a technique for solving that problem. Maity and Roy (2014) discussed a TP with a multi-choice parameter such as; demand, supply and cost, under utility function. A binary variable transformation technique has been used by them to convert multi-choices into a crisp value. In the last goal, programming is used to obtain the optimum solution. Maity and Roy (2016) proposed a model for TP, which has a nonlinear objective (cost) and constraints (demand and supply) in multi-choices. Roy et al. (2012) considered a multi-choice stochastic transportation problem where supply and demand parameter of the constraints followed exponential distribution, and cost coefficients of objective function have multi-choice. A new transformation technique introduced to manipulate cost coefficients of objective function involving multi-choice or goals for binary variables with additional restriction where the additional restriction depend upon the number of aspiration levels associated with each cost coefficient of the objective function. Acharya and Biswal (2016) considered some multi-choice parameters on a MOTP, and interpolating polynomials formulated for the multi-choice parameters. The compromise solution obtained by FP. Kamal et al. (2019) described a bi-level multi-objective production planning problem in which some of the coefficients of objective functions and parameters of constraints are multi-choice. The multi-choices parameters of the prob- 
lem transformed into their equivalent deterministic form based on the binary variables.

The stochastic optimization technique deals with the modelling and optimizing of decision-making problems where probability distributions control the data are known or may be estimated using unknown parameters. SP techniques explain circumstances where random variables identify one or more computational programming problem parameters rather than deterministic variables. While deterministic issues are established with defined parameters, the real-world issues are about the parameters, which are almost probably ambiguous at the time a decision is to be made. If the parameters are unclear but presumed to get into some range of values, a solution can be found that is feasible for all such parameters, which help to optimize a given objective function. In many cases of $\mathrm{TP}$, the parameters have characterized as random variables instead of absolute values. In such TP, the stochastic optimization technique is used to solve the problem. The main concept of solving a stochastic problem is to transform the problem into deterministic form. In the latest years, many investigators have investigated TP's parameters as random variables and have followed various kinds of probability distributions.

Sahoo and Biswal (2005) concerned that SP in which parameters are treated as random variables to capture the uncertainty An approach to deal with uncertainty is assigned a probability distribution to the unknown parameters. Two probability distributions Cauchy distribution and extreme value distribution are introduced for SP, and two different approaches are applied to transform the probabilistic multi-objective LPP into deterministic models. Roy et al. (2012) considered a stochastic transportation problem on which exponential distribution is used to all constraints containing parameters like supply and demand and cost coefficients of objective functions are multi-choice. A new transformation technique is introduced to manipulate cost coefficients of objective function involving multi-choice or goals for binary variables with additional restriction, and the specified probabilistic constraints are transformed into an equivalent deterministic constraint using SP approach. Mahapatra et al. (2013) considered a multi-choice stochastic transportation problem in which the supply and demand parameters of the constraints follow extreme value distribution and some of the cost coefficient of the objective function is multichoice. The probabilistic constraints transformed into deterministic constraints and handled the multi-choice by using binary variables. Roy (2014) presents a multichoice stochastic transportation problem in which supply and demand parameters of the constraints follow Weibull distribution and cost coefficients of the objective function are multi-choice. Barik et al. (2011) considered both single and multi-objective SP problem where the RHS parameter has Pareto distribution with known mean and variance. Chance constrained programming and two-stage SP is used, and FP technique is used to solve the multi-objective model. Biswas and Modak (2011) developed a FGP methodology for solving chance-constrained programming problem involving fuzzy numbers and fuzzy random variables, which follow a standard normal distribution. Barik (2015) presented a linearly constrained probabilistic FGP problem in which the RHS parameters of some constraints follows Pareto distribution with known mean and variance and also simple, weighted, and pre-emptive additive approaches are discussed for probabilistic FGP model. Safi and Ghasemi (2017) described the linear fractional transportation problem with an uncertain parameter with considering the chance-constrained method for the restrictions.

The new development in fuzzy set theory is T1FS, it contains four (minimum) kinds of uncertainties: (1) the definitions of the words used in the precedents and subsequent laws can be unclear, (2) implications can be correlated with a histogram of values, in particular when information is derived from a group of experts who do not all agree, (3) measures that cause a T1FS may be noisy and often uncertain, and (4) data used to fine-tune T1FS parameters can also be noisy. All these types of uncertainties are present in T1FS because membership functions of T1FS are finally in crisp form whereas the T2FS can handle these uncertainties because their membership functions are considered as fuzzy. Membership functions of T2FS are three dimensional that allows some additional degree of freedom to minimize these uncertainties. In other words, the membership functions of T1FS are expressed in absolute numbers, which also have some disadvantage as it has some uncertainties in the membership function. T1FS are not able to directly model such uncertainties because their membership functions are crisp.

On the other hand, T2FS aimed to reduce the level of uncertainty and prompt to get results closer to the degree of truthiness. In other words, T2FS can model such uncertainties in more appropriately since the membership functions are presented in fuzzy nature. Membership functions of T1FS are two-dimensional, whereas membership functions of T2FS are three-dimensional. The third-dimension of T2FS provides additional degrees of freedom, which make possible to directly model uncertainties. To overcome these difficulties of T1FS, Zedah in 1975 suggested the concept of T2FS. In recent years, many experts have utilized the concept of T2FS in decision-making problems. Maity and Roy (2019) developed a new technique for solving TP with T2FS. Sinha et al. (2016) considered a solid TP with profit maximization and time minimization objectives with T2FS and used interactive fuzzy satisficing technique to obtain the optimum solution.

The above discussion on literature and Table 1, reveals that the majority of researchers formulated their work as a single objective or multi-objective and use different fuzzy numbers. MOTP with type 2 fuzzy numbers is very rare. Motivated by such work, we have to try to formulate the TP with multiple objectives in a fuzzy, multi-choice stochastic environment. From Table 1, we have observed that MOTP with type 2 fuzzy numbers in the fuzzy, multichoice stochastic environment is very rare. This is regarded as the big drawback we have found in the past works. 
The above discussion on literature reveals that a lot of research papers have published on MOTP in fuzzy environment. However, the work published on MOAP with T2FS is limited. Table 1 is a list of published works on TP.

\section{Research gap}

After analyzing the above literature reviews of the transportation models, there are still some gaps, which are discussed below:

"»" as per our best knowledge; MOTP with objectives profit maximization and TC minimization with T2FNs is very rare;

Table 1. Review of some existing works

\begin{tabular}{|c|c|c|c|c|c|c|c|c|c|c|c|}
\hline \multirow{3}{*}{ Reference } & \multirow{2}{*}{\multicolumn{3}{|c|}{ Model objective }} & \multicolumn{6}{|c|}{ Nature of uncertainty } & \multirow{3}{*}{\begin{tabular}{|l|} 
Types of fuzzy \\
number / \\
probability \\
distribution
\end{tabular}} & \multirow{3}{*}{ Technique } \\
\hline & & & & \multicolumn{2}{|r|}{ fuzzy } & \multicolumn{2}{|c|}{ stochastic } & \multicolumn{2}{|c|}{ multi-choice } & & \\
\hline & single & multi & survival & $\mathrm{TC}$ & $\mathrm{S}$ and $\mathrm{D}$ & TC & $S$ and $D$ & TC & $S$ and $\mathrm{D}$ & & \\
\hline Bit et al. (1992) & & $\checkmark$ & & & & & & & & & FLP algorithm \\
\hline Li, Lai (2000) & & $\checkmark$ & & & & & & & & & FCP approach \\
\hline El-Wahed (2001) & & $\checkmark$ & & & & & & & & & FP approach \\
\hline Liu (2003) & $\checkmark$ & & & & & & & & & & $\begin{array}{l}\text { lower and upper } \\
\text { bounds for total TC }\end{array}$ \\
\hline $\begin{array}{l}\text { Chakraborty, A., } \\
\text { Chakraborty, M. } \\
(2010)\end{array}$ & & $\checkmark$ & & $\checkmark$ & $\checkmark$ & & & & & interval & $\begin{array}{l}\text { parametric FP and } \\
\text { preemptive GP }\end{array}$ \\
\hline $\begin{array}{l}\text { Dutta, Murthy } \\
(2010)\end{array}$ & $\checkmark$ & & & & $\checkmark$ & & & $\checkmark$ & & trapezoidal & MCGP \\
\hline Roy et al. (2012) & $\checkmark$ & & & & & & $\checkmark$ & $\checkmark$ & & $\begin{array}{l}\text { exponential } \\
\text { distribution }\end{array}$ & multi-choice SP \\
\hline $\begin{array}{l}\text { Mahapatra et al. } \\
(2013)\end{array}$ & $\checkmark$ & & & & & & $\checkmark$ & $\checkmark$ & & $\begin{array}{l}\text { extreme value } \\
\text { distribution }\end{array}$ & $\begin{array}{l}\text { multi-choice } \\
\text { programming }\end{array}$ \\
\hline Maity, Roy (2014) & & $\checkmark$ & & & & & & $\checkmark$ & $\checkmark$ & & GP \\
\hline Roy (2014) & $\checkmark$ & & & & & & $\checkmark$ & $\checkmark$ & & $\begin{array}{l}\text { Weibull } \\
\text { distribution }\end{array}$ & multi-choice SP \\
\hline $\begin{array}{l}\text { Ebrahimnejad } \\
\text { (2014) }\end{array}$ & $\checkmark$ & & & $\checkmark$ & & & & & & $\begin{array}{l}\text { generalised } \\
\text { trapezoidal }\end{array}$ & FTP \\
\hline Liu (2016) & $\checkmark$ & & & $\checkmark$ & $\checkmark$ & & & & & triangular & fractional FTP \\
\hline Maity, Roy (2016) & & $\checkmark$ & & & & & & & $\begin{array}{c}\checkmark \\
\text { D only }\end{array}$ & & BTP \\
\hline Maity et al. (2016) & & $\checkmark$ & & & & $\checkmark$ & $\checkmark$ & & & $\begin{array}{l}\text { normal } \\
\text { distribution }\end{array}$ & FMCGP \\
\hline $\begin{array}{l}\text { Acharya, Biswal } \\
(2016)\end{array}$ & & $\checkmark$ & & & & & & & $\begin{array}{c}\checkmark \\
\text { D only }\end{array}$ & & FP approach \\
\hline Xie et al. (2017) & $\checkmark$ & & & & & & & & & & TPVDS-A algorithm \\
\hline Roy et al. (2017a) & & $\checkmark$ & & & & & & $\checkmark$ & $\checkmark$ & & revised MCGP \\
\hline Roy et al. (2017b) & & $\checkmark$ & & & & & & & $\checkmark$ & & $\begin{array}{l}\text { revised MCGP for } \\
\text { two-stage TP }\end{array}$ \\
\hline $\begin{array}{l}\text { Rani, Gulati } \\
(2017)\end{array}$ & $\checkmark$ & & & $\checkmark$ & $\checkmark$ & & & & & $\begin{array}{l}\text { generalized } \\
\text { exponential } \\
\text { fuzzy number }\end{array}$ & - \\
\hline Roy, Maity (2017) & $\checkmark$ & & & & & & & $\checkmark$ & $\checkmark$ & $\begin{array}{l}\text { multi-choice } \\
\text { interval }\end{array}$ & TP approach \\
\hline $\begin{array}{l}\text { Biswas, Modak } \\
(2017)\end{array}$ & & $\checkmark$ & & & & & & & $\checkmark$ & & FGP \\
\hline Proposed model & & $\checkmark$ & $\checkmark$ & $\checkmark$ & & & $\checkmark$ & & $\checkmark$ & $\begin{array}{l}\text { type } 2 \text { fuzzy } \\
\text { number }\end{array}$ & FGP \\
\hline
\end{tabular}

Notes: TC - transportation cost; S - supply; D - demand.
"» MOTP with T2FNs, where demand and supply are in two situations: (1) multi-choice, and (2) probabilistic random variable is also very rare;

"» a model for MOTP with the rate of increment in TC and rate of decrement in profit on transporting of each product due to the damage, late deliveries, weather conditions, and any other issues have not been discussed in the literature review;

"»" AIC and BIC approach for best-fit probability distributions for MOTP with T2FNs are also infrequent. 


\section{Statement of the transportation problem}

The traditional TP is a well-known problem in our daily life. The main objective behind optimizing and modelling of any TPs under a set of different restrictions to obtain the best solution for the objective function. In many reallife situations problem, the DM wants to ship homogenous kind of products from origins (also called plants, sources, supply points) to different types of destinations (also called sinks, demand points) to satisfy the requirements of the destination fully. In this type of TP, the main aim of the DM to discover the best economic path for transferring a set of the commodity from origin to destination with minimizing the total TC.

\section{Nomenclature}

Indices:

$k$ - index for the objective function, $k=1,2, \ldots, K$;

$i$ - index for the origins item, $i=1,2, \ldots, m$;

$j$ - index for destination items, $j=1,2, \ldots, n$.

Decision variable:

$x_{i j}$ - manufactured units transported from $i$ th origin to $j$ th destination.

\section{Parameters:}

$a_{i}$ - availability at the $i$ th source;

$b_{j}$ - demand at the $j$ th destination;

$c_{i j}$ - transporting cost from $i$ th source to $j$ th destination;

$p_{i j}$ - profit for transporting each unit from $i$ th source to $j$ th destination.

The mathematical model of the transportation problem with equality constraints is defined as:

$$
\begin{aligned}
& \text { Model } 1 \\
& \min Z=\sum_{i=1}^{m} \sum_{j=1}^{n} C_{i j} \cdot x_{i j} \\
& \text { subject to: } \\
& \sum_{j=1}^{n} x_{i j}(\leq,=) a_{i}, i=1,2, \ldots, m ; \\
& \quad \sum_{i=1}^{m} x_{i j}(\geq=) b_{j}, j=1,2, \ldots, n ; \\
& x_{i j} \geq 0, i=1,2, \ldots, m, j=1,2, \ldots, m .
\end{aligned}
$$

where: $m$ is the total number of origins; $n$ is the total number of destinations; $a_{i}$ is the supply of commodity at the $i$ thorigin; $b_{j}$ is the demand for the commodity $j$ th at the destination; $c_{i j}$ is the cost of transportation for a unit quantity of the commodity modify from the ith origin to the $\mathrm{j}$ th destination; $x_{i j}$ is the quantity of the commodity that should be transported from the $i$ th origin to the $j$ th destination to minimize the total TC $Z$.

It is further noted, for the Model 1 (Equation (1)) is feasible if $\sum_{i=1}^{m} a_{i}=\sum_{j=1}^{n} b_{j}$ and reference TP is called balanced transportation problem. Moreover, for the case of inequality constraints Model 1 is feasible if $\sum_{i=1}^{m} a_{i} \geq \sum_{j=1}^{n} b_{j}$ and reference TP is called unbalanced transportation problem.

\section{Transportation problem under T2TpFN}

In TPs, generally, we assume that the transport expenses of items from source to destination are known to the DM. However, in several cases, DM does not know the transport expenses. In such situations, if the transport expenses are not precise and the information is available in vague values. Then the TP can be formulated under fuzziness. In TP, the data cannot be dealt with T1FS because there is much fuzziness involved in the data. Example, TCs will vary from one place to another that might heavily rely on time. Therefore it is necessary to introduce T2FS for attempting to tackle such data.

Here we are formulating the MTOP under type-2 fuzzy numbers. Consider $m$ origins $S_{1}, S_{1}, \ldots, S_{m}, n$ destinations $D_{1}, D_{1}, \ldots, D_{n}$ and $K$ objectives $Z_{1}, Z_{1}, \ldots, Z_{K}$. The aim of the DM is the minimization of all $K$ objectives function. Suppose that the origins $S_{i}$ have a given supply $a_{i}(i=1,2, \ldots, m)$ and destination $D_{j}$ has a required level of demand $b_{j}(j=1,2, \ldots, n)$. Each objective $Z_{K}$ has a penalty cost $c_{i j}^{k}$ while transporting units of the goods from origins $S_{i}$ to destination $D_{j},(i=1,2, \ldots, m ; j=1,2, \ldots, n)$. We extended the Model 1 (Equation (1)) as Model 1a (Equation (2)) by considering the profit cost function with TC objective function. Let suppose in objective functions TC $\tilde{\tilde{c}}_{i j}$ and profit $\tilde{p}_{i j}$ earned on selling the products are T2TpFN. Model 1 can be expressed with vagueness in Model 1a:

\section{Model 1a}

$$
\begin{aligned}
& \max Z_{1}=\sum_{i=1}^{m} \sum_{j=1}^{n} \tilde{\tilde{p}}_{i j} \cdot x_{i j} ; \\
& \min Z_{2}=\sum_{i=1}^{m} \sum_{j=1}^{n} \tilde{\tilde{c}}_{i j} \cdot x_{i j}
\end{aligned}
$$

subject to:

$$
\begin{aligned}
& \sum_{j=1}^{n} x_{i j} \leq \tilde{\tilde{a}}_{i}, i=1,2, \ldots, m ; \\
& \sum_{i=1}^{m} x_{i j} \geq \tilde{\tilde{b}}_{j}, j=1,2, \ldots, n ; \\
& x_{i j} \geq 0, i=1,2, \ldots, m, j=1,2, \ldots, n .
\end{aligned}
$$

The T2TpFN is defuzzified using the technique discussed by Biswas and De (2018). In their proposed work, the T2TpFN is presented in four T1TpFNs, i.e., the four membership function of a T2TpFN are T1TpFNs that constitutes the FOU of the corresponding T2TpFN. Therefore, the defuzzification values of T1TpFNs are used to find the defuzzified value of T2TpFNs. The defuzzification procedure of $\mathrm{T} 2 \mathrm{TpFN}$ is portioned into two-phases. In the Phase 1, the defuzzification technique converts T2TpFN to T1TpFN, and further again (Phase 2) applied to T1TpFNs to obtain the defuzzified value of the T2TpFN. The defuzzification procedure is depicted in Figure 1 Biswas (2018).

Some basic definition of T2TpFN has given below.

\section{Definition 1: T1FS (Biswas (2018))}

A T1FS $\tilde{\lambda}$ has defined on the set of real numbers $Y$ than its membership function $\mu_{\tilde{\lambda}}(x)$ characterized as: 


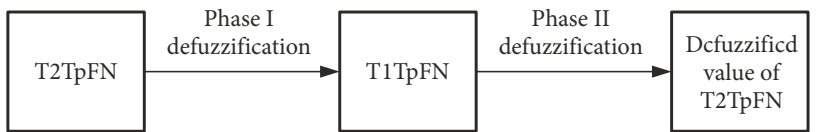

Figure 1. Defuzzification process of T2TpFN

$\mu_{\tilde{\lambda}}: Y \in[0,1] ; 0 \leq \mu_{\tilde{\lambda}}(x) \leq 1$,

where: $y \in Y$, i.e., a T1FS can be defined as: $\tilde{\lambda}=\left\{y, \mu_{\tilde{\lambda}}(x)\right.$ : $y \in Y\}$.

Definition 2: T2FS (Biswas (2018))

Simplification of interval-valued fuzzy sets is known as T2FS. We just considered interval-valued fuzzy sets as fuzzy, and then after the interval-valued fuzzy sets becomes T2FS. Four T1FSs characterized as T2FS, that means the four membership function of a T2FS are T1FSs, the membership function of T2FS is defined in the form:

$$
\mu_{\tilde{\lambda}}: Y \rightarrow \chi[0,1],
$$

where: $\chi[0,1]$ denotes the set of all T1FSs defined on $[0,1]$.

Definition 3: T1TpFN (Biswas (2018))

A fuzzy number $\tilde{\lambda}=\left(x_{1}, x_{2}, x_{3}, x_{4}\right)$ on $R$ is said to be T1TpFN if the following features are shown in membership function:

$$
\mu_{\tilde{\lambda}}(y)= \begin{cases}\frac{y-x_{1}}{x_{2}-x_{1}}, & \text { if } x_{1} \leq y \leq x_{2} \\ 1, & \text { if } x_{2} \leq y \leq x_{3} \\ \frac{x_{4}-y}{x_{4}-x_{3}}, & \text { if } x_{3} \leq y \leq x_{4} \\ 0, & \text { if } y<x_{1}, \text { or } y>x_{4} .\end{cases}
$$

Definition 4: T2TpFN (Biswas (2018))

$\mathrm{T} 2 \mathrm{TpFN}$ can represent in term of four T1TpFN, which are the FOU. Four T1TpFNs are used to represent a T2TpFN. If all T1TpFNs are identical, T2TpFN is termed as normal T2TpFN. T2TpFN is represented as:

$$
\tilde{\tilde{\lambda}}=\left(\tilde{\lambda}_{1}, \tilde{\lambda}_{2}, \tilde{\lambda}_{3}, \tilde{\lambda}_{4}\right) \text {, }
$$

where:

$$
\begin{aligned}
& \tilde{\lambda}_{1}=\left(x_{1}^{L}, x_{0}^{L}, x_{0}^{R}, x_{1}^{R}\right) ; \\
& \tilde{\lambda}_{2}=\left(x_{2}^{L}, x_{0}^{L}, x_{0}^{R}, x_{2}^{R}\right) ; \\
& \tilde{\lambda}_{3}=\left(x_{3}^{L}, x_{0}^{L}, x_{0}^{R}, x_{3}^{R}\right) ; \\
& \tilde{\lambda}_{4}=\left(x_{4}^{L}, x_{0}^{L}, x_{0}^{R}, x_{4}^{R}\right)
\end{aligned}
$$

represents T1TpFNs.

Membership values of T1TpFNs must hold the relation:

$$
\mu_{\tilde{\lambda}_{1}}(y) \leq \mu_{\tilde{\lambda}_{2}}(y) \leq \mu_{\tilde{\lambda}_{3}}(y) \leq \mu_{\tilde{\lambda}_{4}}(y) .
$$
$\mu_{\tilde{\lambda}}(y)=\left\{\begin{array}{ll}\left(\mu_{1 j}^{L}(y), \mu_{2 j}^{L}(y), \mu_{3 j}^{L}(y), \mu_{4 j}^{L}(y)\right) & \text { if } x_{j+1}^{L} \leq y \leq x_{j}^{L} ; \\ \left(\hat{\mu}_{1 j}^{R}(y), \hat{\mu}_{2 j}^{R}(y), \hat{\mu}_{3 j}^{R}(y), \hat{\mu}_{4 j}^{R}(y)\right) & \text { if } x_{j}^{R} \leq y \leq x_{j+1}^{R} ; \\ 0, & \text { otherwise, }\end{array}\right.$;

Primary membership function $\mu_{\tilde{\lambda}}(y)$ of the T2TpFN where:

$$
\begin{aligned}
& \mu_{i j}^{L}(y)= \begin{cases}\frac{y-x_{i}^{L}}{x_{0}^{L}-x_{i}^{L}}, & \text { if } i>j ; \\
0, & \text { if } i \leq j,\end{cases} \\
& j=0,1,2,3 ; \\
& i=1,2,3,4
\end{aligned}
$$

and

$$
\begin{aligned}
& \hat{\mu}_{i j}^{R}(y)= \begin{cases}\frac{x_{i}^{R}-y}{x_{i}^{R}-x_{0}^{R}}, & \text { if } i>j ; \\
0, & \text { if } i \leq j,\end{cases} \\
& j=0,1,2,3 ; \\
& i=1,2,3,4 .
\end{aligned}
$$
is:

Secondary membership function $\gamma_{\mu} \tilde{\tilde{\lambda}}(\mu)$ for T2TpFN

$$
\begin{aligned}
& \gamma_{\mu_{\lambda}}(\mu)= \begin{cases}\frac{\mu-\mu_{1 j}^{L}(y)}{\mu_{2 j}^{L}(y)-\mu_{1 j}^{L}(y)}, & \text { if } \mu_{1 j}^{L}(y) \leq \mu \leq \mu_{2 j}^{L}(y) ; \\
1, & \text { if } \mu_{2 j}^{L}(y) \leq \mu \leq \mu_{3 j}^{L}(y) ; \\
\frac{\mu_{4 j}^{L}(y)-\mu}{\mu_{4 j}^{L}(y)-\mu_{3 j}^{L}(y)}, & \text { if } \mu_{3 j}^{L}(y) \leq \mu \leq \mu_{4 j}^{L}(y) ; \\
0, & \text { otherwise; }\end{cases} \\
& x_{4}^{L} \leq y \leq x_{0}^{L}
\end{aligned}
$$

and

$$
\begin{aligned}
& \gamma_{\mu_{\lambda}}(\mu)= \begin{cases}\frac{\mu-\hat{\mu}_{1 j}^{R}(y)}{\hat{\mu}_{2 j}^{R}(y)-\hat{\mu}_{1 j}^{R}(y)}, & \text { if } \hat{\mu}_{1 j}^{R}(y) \leq \mu \leq \hat{\mu}_{2 j}^{R}(y) ; \\
1, & \text { if } \hat{\mu}_{2 j}^{R}(y) \leq \mu \leq \hat{\mu}_{3 j}^{R}(y) ; \\
\frac{\hat{\mu}_{4 j}^{R}(y)-\mu}{\hat{\mu}_{4 j}^{R}(y)-\hat{\mu}_{3 j}^{R}(y)}, & \text { if } \hat{\mu}_{3 j}^{R}(y) \leq \mu \leq \hat{\mu}_{4 j}^{R}(y) ; \\
0, & \text { otherwise; }\end{cases} \\
& x_{0}^{R} \leq y \leq x_{4}^{R} .
\end{aligned}
$$

Now using the above-given definitions, we applied the concept on the TP Model 1a (Equation (2)). In this model, we have assumed that cost function is objective function is T2TpFN. The two-phase defuzzification procedure is given in brief.

\section{Phase 1}

Let:

$$
\tilde{\tilde{C}}_{i j}=\left(\tilde{C}_{i j 1}, \tilde{C}_{i j 2}, \tilde{C}_{i j 3}, \tilde{C}_{i j 4}\right)
$$

be a $\mathrm{T} 2 \mathrm{TpFN}$,

where each

$$
\tilde{C}_{i j}=\left(C_{i j l}^{L}, C_{i j 0}^{L}, C_{i j 0}^{R}, C_{i j l}^{R}\right), l=1,2,3,4 .
$$

In this phase we have presented $\mathrm{T} 2 \mathrm{TpFN}$ into four T1TpFN, i.e.:

$$
V\left(\tilde{\tilde{C}}_{i j}\right)=\left(V\left(\tilde{C}_{i j 1}\right), V\left(\tilde{C}_{i j 2}\right), V\left(\tilde{C}_{i j 3}\right), V\left(\tilde{C}_{i j 4}\right)\right) ;
$$




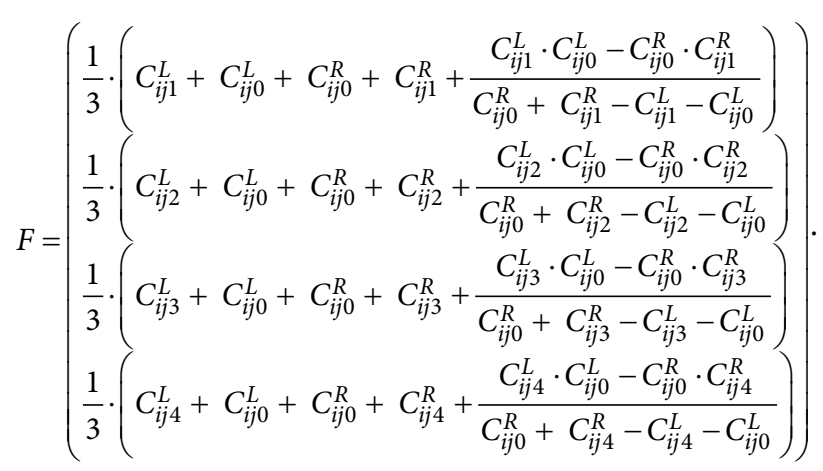

\section{Phase 2}

The resultant T1TpFN is again defuzzified to obtain a crisp value of T2TpFN.

Let $V\left(\tilde{\tilde{C}}_{i j}\right)$ is T1TpFN, which is also again fuzzy numbers, and with the help of these fuzzy numbers, we obtain a crisp value using the formula:

$$
\begin{aligned}
& D V\left(\tilde{\tilde{C}}_{i j}\right)=\frac{1}{3} \cdot\left(V\left(\tilde{C}_{i j 1}\right)+V\left(\tilde{C}_{i j 2}\right)+V\left(\tilde{C}_{i j 3}\right)+V\left(\tilde{C}_{i j 4}\right)+\right. \\
& \left.\frac{V\left(\tilde{C}_{i j 1}\right) \cdot V\left(\tilde{C}_{i j 2}\right)-V\left(\tilde{C}_{i j 3}\right) \cdot V\left(\tilde{C}_{i j 4}\right)}{V\left(\tilde{C}_{i j 3}\right)+V\left(\tilde{C}_{i j 4}\right)-V\left(\tilde{C}_{i j 1}\right)-V\left(\tilde{C}_{i j 2}\right)}\right) ; \\
& D V\left(\tilde{\tilde{P}}_{i j}\right)=\frac{1}{3} \cdot V\left(\tilde{P}_{i j 1}\right)+V\left(\tilde{P}_{i j 2}\right)+V\left(\tilde{P}_{i j 3}\right)+V\left(\tilde{P}_{i j 4}\right)+ \\
& \left.\frac{V\left(\tilde{P}_{i j 1}\right) \cdot V\left(\tilde{P}_{i j 2}\right)-V\left(\tilde{P}_{i j 3}\right) V\left(\tilde{P}_{i j 4}\right)}{V\left(\tilde{P}_{i j 3}\right)+V\left(\tilde{P}_{i j 3}\right)-V\left(\tilde{P}_{i j 1}\right)-V\left(\tilde{P}_{i j 2}\right)}\right)
\end{aligned}
$$

Similarly, the same procedure is followed for $\tilde{\tilde{a}}_{i}$ and $\tilde{\tilde{b}}_{i}$ T2TpFN to obtain the crisp value, i.e.:

$$
\begin{aligned}
& D V\left(\tilde{\tilde{a}}_{i}\right)=\frac{1}{3} \cdot\left(V\left(\tilde{a}_{i 1}\right)+V\left(\tilde{a}_{i 2}\right)+V\left(\tilde{a}_{i 3}\right)+V\left(\tilde{a}_{i 4}\right)+\right. \\
& \left.\frac{V\left(\tilde{a}_{i 1}\right) \cdot V\left(\tilde{a}_{i 2}\right)-V\left(\tilde{a}_{i 3}\right) \cdot V\left(\tilde{a}_{i 4}\right)}{V\left(\tilde{a}_{i 3}\right)+V\left(\tilde{a}_{i 4}\right)-V\left(\tilde{a}_{i 1}\right)-V\left(\tilde{a}_{i 2}\right)}\right) \\
& D V\left(\tilde{\tilde{b}}_{j}\right)=\frac{1}{3} \cdot\left(V\left(\tilde{b}_{j 1}\right)+V\left(\tilde{b}_{j 2}\right)+V\left(\tilde{b}_{j 3}\right)+V\left(\tilde{b}_{j 4}\right)+\right. \\
& \left.\frac{V\left(\tilde{b}_{j 1}\right) \cdot V\left(\tilde{b}_{j 2}\right)-V\left(\tilde{b}_{j 3}\right) \cdot V\left(\tilde{b}_{j 4}\right)}{V\left(\tilde{b}_{j 3}\right)+V\left(\tilde{b}_{j 4}\right)-V\left(\tilde{b}_{j 1}\right)-V\left(\tilde{b}_{j 2}\right)}\right)
\end{aligned}
$$

\section{TC and profit with increment and decrement rate function}

In general, the primary goal of the $\mathrm{DM}$ in the TP is to ship the products from sources to destinations should have been finished within the defined period. However, late delivery in TP is phenomenal due to some random factors such as weather conditions, road transport, traffic, etc. In such cases, some different types of penalty expenses may happen to owe to the delay or cancellation of the ordered from the customers. There are so many uncontrolled factors responsible, which can influence product delay delivery such as road circumstances, traffic, weather, etc. Thus these types of penalty expenses can influence the company goodwill. Therefore, in these circumstances, profit or transportation expenses may not be regarded as a fixed amount, i.e. the profit or expenses are inherently unsure. We also considered the concept of "survival cost/ profit" for such instant.

The likelihood of completing the shipment of products from sources to locations within the speculated period without failing due to any reason for shipment of products is known as cost reliability, due to all we may have a probabilistic price in the TP. If the probabilistic price happens in the transportation problem, the initial value of the products may increase, and therefore the profit will be suffered.

Let $\theta_{i j}$ be the rate of depreciation in profit due to damage units, late delivery, etc. the rate of depreciation in profit is calculated as follows:

$$
\theta_{i j}=\frac{N_{i}(t)-N_{j}(t+\delta \cdot t)}{N_{i}(t)},
$$

where: $N_{i}(t)$ is the total number of items, which is transported to the $j$ th destination from the $i$ th origin.

Now, we have considered cost and profit as a function of time. Then, the cost and profit objective functions of $\mathrm{TP}$ are considered as survival cost and profit. $N_{j}(t+\delta \cdot t)$ is the items remain in good condition at the final destination. Hence the profit suffered by the depreciation rate $\theta_{i j} \cdot \tilde{\tilde{p}}_{i j}$. The total profit is calculated as:

$$
\text { total profit }=D V\left(\tilde{\tilde{p}}_{i j}\right)-\theta_{i j} \cdot\left(D V\left(\tilde{\tilde{p}}_{i j}\right)\right) .
$$

Further, we assumed that the TC would also be increased due to uncontrolled randomly occurred factors occurred during the transportation. These uncontrolled factors might be a minor accident or major accident, traffic and other similar issues. Let $\gamma_{i j}$ be the rate of increment in TP cost, which is calculated as follows:

$$
\gamma_{i j}=\frac{C_{i j}(t+\delta t)-C_{i j}(t)}{C_{i j}(t)},
$$

where: $C_{i j}(t)$ is the total cost of transportation from origin to destination, and $C_{i j}(t+\delta t)$ is the cost with increment due to traffic and other anonymous issues. The total $T C$ is calculated as:

$$
\text { total } T C=D V\left(\tilde{\tilde{C}}_{i j}\right)+\gamma_{i j} \cdot\left(D V\left(\tilde{\tilde{C}}_{i j}\right)\right)
$$

After including the incremental rate in TP cost function and depreciation rate in profit function, we have Model 1b (Equation (17)).

$$
\begin{aligned}
& \text { Model 1b } \\
& \begin{aligned}
& \max Z_{1}=\sum_{i=1}^{m} \sum_{j=1}^{n}\left(D V\left(\tilde{\tilde{p}}_{i j}\right)-\theta_{i j} \cdot\left(D V\left(\tilde{\tilde{p}}_{i j}\right)\right)\right) \cdot x_{i j} ; \\
& \min Z_{2}=\sum_{i=1}^{m} \sum_{j=1}^{n}\left(D V\left(\tilde{\tilde{C}}_{i j}\right)+\gamma_{i j} \cdot\left(D V\left(\tilde{\tilde{C}}_{i j}\right)\right)\right) \cdot x_{i j} \\
& \text { subject to: } \\
& \sum_{j=1}^{n} x_{i j} \leq D V\left(\tilde{\tilde{a}}_{i}\right), i=1,2, \ldots, m ;
\end{aligned}
\end{aligned}
$$




$$
\begin{aligned}
& \sum_{i=1}^{m} x_{i j} \geq D V\left(\tilde{\tilde{b}}_{j}\right), j=1,2, \ldots, n \\
& x_{i j} \geq 0, i=1,2, \ldots, m, j=1,2, \ldots, n .
\end{aligned}
$$

\section{Mixed uncertain constraints of TP}

Consider a case where the right side of the constraints are imprecise, and the multi-choice parameter and probabilistic random variable in the $\mathrm{TP}$ reflect this vagueness on the right side of the constraint. Nevertheless, the objective function of uncertainty is assumed as T2TpFN.

\subsection{TP with demand and supply are interval type multi-choice}

There are some real-life situations where, the parameters of the TPs like demand, and supply problem are multichoices. Then our new model with demand and supply in multi-choice is described as:

\section{Model 1c}

$$
\begin{aligned}
& \max Z_{1}=\sum_{i=1}^{m} \sum_{j=1}^{n}\left(D V\left(\tilde{\tilde{p}}_{i j}\right)-\theta_{i j} \cdot\left(D V\left(\tilde{\tilde{p}}_{i j}\right)\right)\right) \cdot x_{i j} \\
& \min Z_{2}=\sum_{i=1}^{m} \sum_{j=1}^{n}\left(D V\left(\tilde{\tilde{C}}_{i j}\right)+\gamma_{i j} \cdot\left(D V\left(\tilde{\tilde{C}}_{i j}\right)\right)\right) \cdot x_{i j}
\end{aligned}
$$

subject to:

$$
\begin{aligned}
& \sum_{j=1}^{n} x_{i j} \leq a_{i}^{1} \text { or } a_{i}^{2} \text { or...or } a_{i}^{p}, i=1,2, \ldots, m ; \\
& \sum_{i=1}^{m} x_{i j} \geq b_{j}^{1} \text { or } b_{j}^{2} \text { or } \ldots \text { or } b_{j}^{q}, j=1,2, \ldots, n ; \\
& x_{i j} \geq 0, i=1,2, \ldots, m, j=1,2, \ldots, n .
\end{aligned}
$$

The multi-choices is dealt with using the technique of Roy and Maity (2017):

$$
\sum_{j=1}^{n} x_{i j} \leq a_{i}^{1}
$$

or

$$
\begin{aligned}
& a_{i}^{2} \text { or...or } a_{i}^{p}, \\
& i=1,2, \ldots, m= \\
& \sum_{k=1}^{K}(\text { term })^{k} \cdot\left(a_{i}^{k^{l}}\left(1-\lambda^{a_{i}^{k}}\right)\right)+a_{i}^{k^{u}} \cdot \lambda^{a_{i}^{k}} \\
& \sum_{i=1}^{m} x_{i j} \geq b_{j}^{1}
\end{aligned}
$$

or

$$
\begin{aligned}
& b_{j}^{2} \text { or ....or } b_{j}^{q}, \\
& j=1,2, \ldots, n= \\
& \sum_{k=1}^{K}(\text { term })^{k} \cdot b_{j}^{k^{l}} \cdot 1-\lambda^{b_{j}^{k}}+b_{j}^{k^{u}} \lambda^{b_{j}^{k}} .
\end{aligned}
$$

\subsection{TP with demand and supply} are probabilistic random variables

\section{Model 1d}

$$
\begin{aligned}
& \max Z_{1}=\sum_{i=1}^{m} \sum_{j=1}^{n}\left(D V\left(\tilde{\tilde{p}}_{i j}\right)-\theta_{i j} \cdot D V\left(\tilde{\tilde{p}}_{i j}\right)\right) \cdot x_{i j} ; \\
& \min Z_{2}=\sum_{i=1}^{m} \sum_{j=1}^{n}\left(D V\left(\tilde{\tilde{C}}_{i j}\right)+\gamma_{i j} \cdot\left(D V\left(\tilde{\tilde{C}}_{i j}\right)\right)\right) x_{i j}
\end{aligned}
$$

subject to:

$$
\begin{aligned}
& \operatorname{Prob}\left(\sum_{j=1}^{n} x_{i j} \leq a_{i}\right) \geq \phi_{i}, i=1,2, \ldots, m ; \\
& \operatorname{Prob}\left(\sum_{i=1}^{m} x_{i j} \geq b_{j}\right) \geq \psi_{j}, j=1,2, \ldots, n ; \\
& x_{i j} \geq 0, i=1,2, \ldots, m, j=1,2, \ldots, n .
\end{aligned}
$$

where: $0 \leq \phi_{i} \leq 1$ and $0 \leq \psi_{j} \leq 1, \forall i, j$ are the specified probability limits of the stochastic constraints.

In Model 1d (Equation (21)) is reflecting a condition where the constraints are in stochastic form. The probabilistic random parameters of TPs are assumed and followed some particular distribution. Moreover, the parameters of TP followed several distributions, which have shown in Table 2. Table 2 Provide a summary of probability distributions and their parameters estimation, equivalent deterministic form and feasibility conditions. Finally, the best fit of a probability distribution model for the probabilistic random parameters is approximated using AIC and BIC. Several authors have worked on the same concept. We have given a summary table of their works, which is given below.

\subsection{AIC and BIC approach for validation of best fit}

Model selection problems belong to the process of choosing the best model from the set of models using the available data. The most common techniques used for finding the best-fitted models on a data set are AIC and BIC. Akaike (1974) proposed the concept of AIC; the bestfitting probability distribution is selected based on the criteria of minimizes the negative likelihood in addition to a penalty term described by the number of parameters. Apart from AIC, there is another criterion known as BIC, which revolves around the Bayesian system. Stone (1979) proposed the concept of BIC for best fit. The concept is similar to AIC, that is to minimize the likelihood. BIC is constructed from a Bayesian point of view, to find the most likely model fitting the data. AIC and BIC have the same goal of selecting the best models. These two techniques are known as penalized likelihood methods. The formulas are given below:

$$
\begin{aligned}
& A I C=-2 \cdot \log p(\log \text { likelihood })+2 \cdot p ; \\
& B I C=-2 \cdot \log p(\log \text { likelihood })+p \cdot \log (n),
\end{aligned}
$$

where: $p$ is the number of estimated parameters in the distribution; $n$ is the total sample size. 


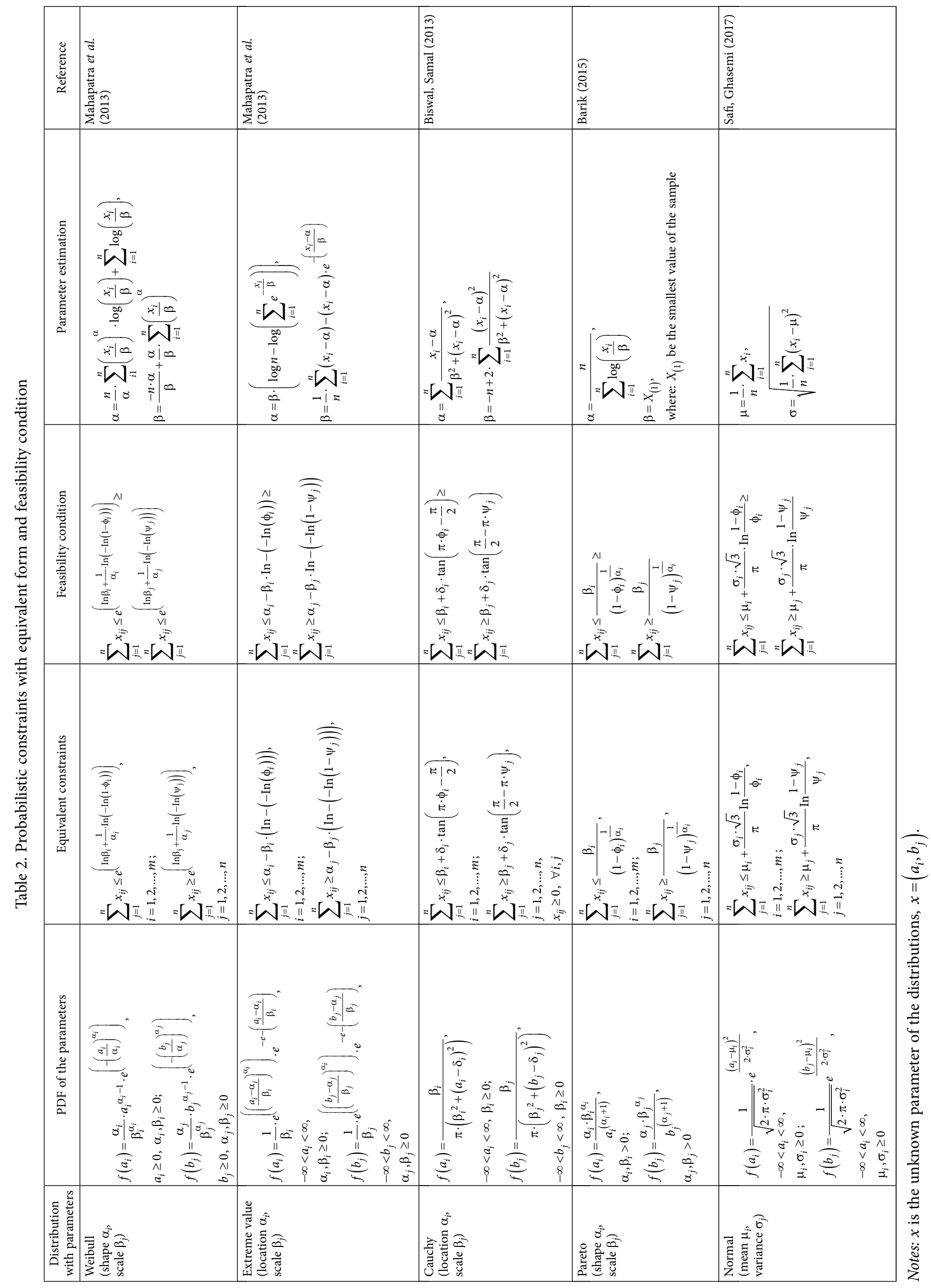




\section{Stepwise solution procedure}

FGP is a versatile and robust method for dealing the multi-objective decision-making problems. Therefore, we also have used to solve our formulated TP.

The stepwise procedure for solving the TP is given as follows.

Step 1. Analyze the problem, is the problem crisp, if yes; go to Step 3. Otherwise, convert the problem into a crisp problem.

Step 2. The appropriate defuzzification technique is used to convert uncertain values into their equivalent crisp form.

Step 3. Solve the various objective optimization problems by considering one objective at the moment and ignoring the other with the same set of constraints. The resulting solution is the idle solution. Idle solutions use to construct the payoff matrix. Finally, the payoff matrix enables each objective function to build the aspiration level.

Step 4 . The aspiration level of the $k$ th objective function is set as the goal value $g_{k}, k=1,2$.

Find $X=\left(x_{1}, x_{2}, \ldots, x_{n}\right)$ to optimize the following fuzzy goals:

$$
\begin{aligned}
& Z_{1}(X) \succeq g_{1} ; \\
& Z_{2}(X) \preceq g_{2}
\end{aligned}
$$

subject to the set of constraints $x \in X$,

where: $g_{1}=\max \left(Z_{1}(x)\right)$ and $g_{2}=\min \left(Z_{2}(x)\right)$. The symbol " $\succeq$ " (the type of fuzzy-max) referring to that $Z_{1}(X)$ should be approximately greater than or equal to the aspiration level $g_{1}$ up to the specified tolerance limit. The symbol " $\preceq$ " (the type of fuzzy-min) referring to that $Z_{2}(X)$ should be approximately less than or equal to the aspiration level $g_{2}$ up to the specified tolerance limit.

Step 5. Construct the fuzzy linear membership function for the fuzzy goal of:

$$
\mu= \begin{cases}1, & \text { if } Z_{1}(X) \geq g_{1} ; \\ \frac{Z_{1}(X)-L_{1}}{g_{1}-L_{1}}, & \text { if } L_{1} \leq Z_{1}(X) \leq g_{1} \\ 0, & \text { if } Z_{1}(X) \leq L_{1},\end{cases}
$$

where: $L_{1}$ is the lower tolerance limit.

Similarly, the membership function for the fuzzy goal $Z_{2} \preceq g_{2}$ :

$$
\mu= \begin{cases}1, & \text { if } Z_{2}(X) \leq g_{2} ; \\ \frac{U_{2}-Z_{2}(X)}{U_{2}-g_{2}}, & \text { if } g_{2} \leq Z_{2}(X) \leq U_{2} ; \\ 0, & \text { if } Z_{2}(X) \geq U_{2},\end{cases}
$$

where: $U_{2}$ is the upper tolerance limit.

Step 6. Finally, following all the above-given steps, the resultant mathematical form is:

$$
\max D(\mu)=\mu
$$

subject to set constraint $x \in X$

and some additional constraints:

$$
\begin{aligned}
& \mu=\frac{Z_{1}(X)-L_{1}}{g_{1}-L_{1}} ; \\
& \mu=\frac{U_{2}-Z_{2}(X)}{U_{2}-g_{2}} ; \\
& 0 \leq \mu \leq 1,
\end{aligned}
$$

where: $D(\mu)$ is a fuzzy achievement function. Finally, we changed the multi-objective decision-making problems into a single objective optimization problem that can be solved using an appropriate classical optimization technique.

\section{Illustrative case study}

We considered a MOTP to verify the efficiency and usefulness of the suggested work. A transportation company intends to transport its different kinds of perishable and sensitive to damage items, etc, from three states: Uttar Pradesh (a1), Uttarakhand (a2) and Punjab (a3), to three districts of Kashmir: Srinagar (b1), Anantnag (b2) and Baramula (b3). The items are transported in these locations for the first time. Therefore, DM no data of previous years. Nevertheless, from experience and his expert market analysis, he has some incomplete information. In this TP, we have considered two objectives, first objective $Z_{1}$ is to maximize the total profit and second objective $Z_{2}$ is to minimize the total TC.

In the real-world problems, several diverse situations occur such as uncertain judgments, unpredictable conditions or human error, incomplete knowledge and information, seasonality, change in policy by the governments, etc., due to which we do not need to get a relevant, precise data about the problem. These discussed factors that lead to uncertainty in the parameters of the TPs. Such type of impreciseness of any data can be represented in different ways. To present this uncertainty in our proposed model, we have discussed T2TpFN, multi-choice approach and probabilistic random variables. In our proposed model, we have considered the profit and TC of MOTP with vagueness. T2TpFN describes this vagueness.

Let suppose that the profit on delivering items from $i$ th source to $j$ th destinations and the TC of delivering the items is available in the form T2TpFNs. Table 3 is for instant information available to the DM.

The two-phase defuzzification procedure, which is defined in Equations (9) and (10) has been used to obtain the crisp form of T2TpFNs. The resultant crisp values of Phases 1 and 2 are summarized in Table 4 .

In the proposed work, the objective function will remain in the form of T2TpFN for the other three cases. The other three cases are given below.

\section{Case 1: when both constraints (demand and supply) are presented by T2TpFN}

In our proposed model, we have considered the demand and supply of the MOTP with vagueness. 
Table 3. Imprecise profit and TC

\begin{tabular}{|c|c|}
\hline \multicolumn{2}{|c|}{$Z_{1}$} \\
\hline$\tilde{\tilde{p}}_{i j}$ & $\tilde{\tilde{c}}_{i j}$ \\
\hline$(68,70,72,76)$ & $(498,500,503,507)$ \\
$(66,70,72,78)$ & $(497,500,503,509)$ \\
$(63,70,72,82)$ & $(495,500,503,512)$ \\
$(60,70,72,85)$ & $(492,500,503,514)$ \\
\hline$(78,80,83,88)$ & $(598,600,602,606)$ \\
$(75,80,83,85)$ & $(595,600,602,608)$ \\
$(74,80,83,91)$ & $(594,600,602,611)$ \\
$(71,80,83,94)$ & $(592,600,602,613)$ \\
\hline$(76,78,80,83)$ & $(618,620,622,625)$ \\
$(74,80,83,91)$ & $(595,600,622,627)$ \\
$(73,74,80,85)$ & $(613,620,622,631)$ \\
$(70,78,80,87)$ & $(610,620,622,635)$ \\
\hline$(78,80,83,87)$ & $(598,600,602,606)$ \\
$(75,80,83,88)$ & $(595,600,602,608)$ \\
$(74,80,83,91)$ & $(594,600,602,611)$ \\
$(71,80,83,94)$ & $(592,600,602,613)$ \\
\hline$(70,72,73,77)$ & $(498,500,503,507)$ \\
$(68,72,73,81)$ & $(497,500,503,509)$ \\
$(65,72,73,81)$ & $(495,500,503,512)$ \\
$(64,72,73,84)$ & $(492,500,503,514)$ \\
\hline
\end{tabular}

\begin{tabular}{|c|c|}
\hline \multicolumn{2}{|c|}{$Z_{1}$} \\
\hline$\tilde{\tilde{p}}_{i j}$ & $\tilde{\tilde{c}}_{i j}$ \\
\hline$(82,84,87,91)$ & $(548,550,551,554)$ \\
$(80,84,87,91)$ & $(545,550,551,556)$ \\
$(77,84,87,93)$ & $(543,550,551,559)$ \\
$(76,84,87,96)$ & $(540,550,551,563)$ \\
\hline$(88,90,91,94)$ & $(648,650,652,655)$ \\
$(87,90,91,96)$ & $(642,650,652,657)$ \\
$(84,90,91,99)$ & $(640,650,652,660)$ \\
$(81,90,91101)$ & $(637,650,652,664)$ \\
\hline$(78,80,83,87)$ & $(598,600,602,606)$ \\
$(75,80,83,88)$ & $(595,600,602,608)$ \\
$(74,80,83,91)$ & $(594,600,602,611)$ \\
$(71,80,83,94)$ & $(592,600,602,613)$ \\
\hline$(74,76,79,80)$ & $(578,580,581,584)$ \\
$(73,76,79,83)$ & $(575,580,581,586)$ \\
$(71,76,79,86)$ & $(573,580,581,604)$ \\
$(70,76,79,88)$ & $(570,580,581,604)$ \\
\hline
\end{tabular}

Table 4. Defuzzifucition Phases 1 and 2 crisp values of profit and TC

\begin{tabular}{|c|c|c|c|}
\hline \multicolumn{2}{|c|}{$Z_{1}$} & $D V\left(\tilde{\tilde{c}}_{i j}\right)$ \\
\hline$V\left(\tilde{\tilde{p}}_{i j}\right)$ & $D\left(\tilde{\tilde{p}}_{i j}\right)$ & $V\left(\tilde{\tilde{c}}_{i j}\right)$ & 502.30 \\
\hline$(72.06,72.16,71.80,71.50)$ & 71.87 & $(502.08,502.40,502.73,502.44)$ & 601.69 \\
\hline$(82.08,81.50,82.11,82.12)$ & 81.90 & $(601.60,601.31,601.94,601.95)$ & 621.30 \\
\hline$(79.29,78.40,78.11,78.68)$ & 78.66 & $(621.29,621.40,621.60,621.31)$ & 601.69 \\
\hline$(82.08,81.50,82.11,82.12)$ & 81.90 & $(601.60,601.31,601.94,601.95)$ & 502.30 \\
\hline$(73.12,72.81,72.82,73.47)$ & 73.13 & $(502.08,502.40,502.73,502.44)$ & 550.83 \\
\hline$(84.88,85.50,85.19,85.81)$ & 85.34 & $(651.29,650.05,650.36,650.67)$ & 650.98 \\
\hline$(90.80,91.13,91.14,90.50)$ & 90.80 & $(601.60,601.31,601.94,601.95)$ & 601.69 \\
\hline$(82.08,81.50,82.11,82.12)$ & 81.90 & $(580.80,580.50,580.80,583.77)$ & 581.29 \\
\hline$(77.20,77.70,78.11,78.42)$ & 77.84 & & \\
\hline
\end{tabular}

Let suppose that the demand and supply of the TP are available in the form T2TpFNs. Table 5 is for instant information available to the DM.

The two-phase defuzzification procedure, which is defined in Equations (11) and (12) has been used to obtain the crisp form of T2TpFNs. The resultant crisp values of Phases 1 and 2 are summarised in Table 6.

In order, to obtain the lower and upper bound (pay off matrix) of each objective functions; we solved each objective separately under the same set of constraints by using optimization software LINGO 16.0. The values are given below:

$$
\begin{aligned}
& 49752.43 \leq Z_{1} \leq 64875.59 \\
& 2878296.0 \leq Z_{2} \leq 3708667.0
\end{aligned}
$$

In this case, we have considered the constraints (demand and supply) of the formulated model of MOTP are uncertain. However, somehow, we figure out the range of constraints. T2TpFN used to presents this uncertainty. After following the stepwise procedure discussed in Section 5, we obtained the compromise solution. The discussed model is solved by using optimization software LINGO 16.0 (https://lingo.informer.com).

T2TpFN:

$Z_{1}=57564.71$,

$x_{11}=4$,

$x_{12}=73$,

$x_{13}=735$,

$x_{22}=913$;

$Z_{2}=3279763$,

$x_{23}=0$,

$x_{31}=922$,

$x_{32}=0$,

$x_{33}=407$. 


\section{Case 2: when the constraints are multi-choice}

In several real-life situations where parameters of decision-making problems have multiple choices. In those situations where DMs are allowed to set a goal of multiple choices. Let suppose that the demand and supply of the TP are available in the form multi-choice. Table 7. is for instant information available to the DM.

After using Equations (19) and (20) the multi-choice parameter is converted into a crisp value.

In order, to obtain the lower and upper bound (pay off matrix) of each objective function, we solved each objective separately under the same set of constraints by using optimization software LINGO 16.0. The values are given below:

$$
\begin{aligned}
& 33829.06 \leq Z_{1} \leq 68169.10 \\
& 1959488 \leq Z_{2} \leq 3476456
\end{aligned}
$$

In this case, we have also considered the constraints (demand and supply) of the formulated model of MOTP are uncertain. Values of the parameters (demand and supply) are available in multiple choices to us, and we do not know, which value is a better fit for getting the optimal solutions. This type of uncertainty is presented by multichoice approach. After following the stepwise procedure discussed in Section 5, we obtained the compromise solution. The discussed model is solved by using optimization software LINGO 16.0.

Multi-choice:

$$
\begin{aligned}
& Z_{1}=51555.71, \\
& Z_{2}=2693496 ; \\
& x_{11}=2, \\
& x_{12}=0, \\
& x_{13}=0, \\
& x_{21}=0, \\
& x_{22}=1100, \\
& x_{23}=0, \\
& x_{31}=713, \\
& x_{32}=0, \\
& x_{33}=800 .
\end{aligned}
$$

The values of the rate of increment and decrement are obtained by using Equations (13)-(16). Table 8 shows the depreciation rate in profit and increment rate in TC.

Table 5. Imprecise demand and supply

\begin{tabular}{|c|c|}
\hline Supply $a_{i}$ & Demand $b_{j}$ \\
\hline$(1000,1100,1200,1300)$ & $(800,900,950,1000)$ \\
$(980,1100,1200,1350)$ & $(780,900,950,1050)$ \\
$(900,1100,1200,1380)$ & $(750,900,950,1080)$ \\
$(800,1100,1200,1400)$ & $(700,900,950,1100)$ \\
\hline$(800,900,950,1000)$ & $(800,900,1100,1200)$ \\
$(780,900,950,1050)$ & $(780,900,1100,1250)$ \\
$(750,900,950,1080)$ & $(720,900,1100,1300)$ \\
$(700,900,950,1100)$ & $(610,900,1100,1400)$ \\
\hline$(1100,1300,1400,1550)$ & $(1000,1100,1200,1300)$ \\
$(1050,1300,1400,1600)$ & $(980,1100,1200,1350)$ \\
$(1000,1300,1400,1650)$ & $(900,1100,1200,1380)$ \\
\hline
\end{tabular}

Table 6. Defuzzifucition of the T2TFN with T1TFN and crisp value of demand and supply

\begin{tabular}{|c|c|c|c|}
\hline$V\left(\tilde{\tilde{a}}_{i}\right)$ & $D\left(\tilde{\tilde{a}}_{i}\right)$ & $V\left(\tilde{\tilde{\sigma}}_{j}\right)$ & \\
\hline$(1150,1158.95,1143.91,1119.05)$ & 1142 & $(910,918.85,918.77,909.25)$ & 912.98 \\
\hline$(910,918.85,918.77,909.25)$ & 912.98 & $(1000,1008.51,1005.81,1003)$ & 986.40 \\
\hline$(1334.85,1334.62,1334.44,1318.52)$ & 1329.33 & $(1150,1158.95,1143.91,1119.05)$ & 1142 \\
\hline
\end{tabular}

Table 7. Summarises the value of demand and supply with interval type multi-choice

\begin{tabular}{|l|l|}
\hline$a_{1}=(800,1000),(1000,1200),(1200,1400)$ & $b_{1}=(700,900),(900,1100)$ \\
\hline$a_{2}=(700,900),(900,1100)$ & $b_{2}=(600,800),(800,1000),(1000,1200),(1200,1400)$ \\
\hline$a_{3}=(900,1100),(1100,1300),(1300,1500),(1500,1700)$ & $b_{3}=(800,1000),(1000,1200),(1200,1400)$ \\
\hline
\end{tabular}

Table 8. Depreciation rate in profit and increment rate in TC

\begin{tabular}{|l|l|l|l|l|l|l|l|l|l|l|}
\hline$\theta_{i j}$ & 0.20 & 0.17 & 0.17 & 0.23 & 0.30 & 0.15 & 0.25 & 0.10 & 0.18 \\
\hline$\gamma_{i j}$ & 0.30 & 0.15 & 0.18 & 0.29 & 0.13 & 0.21 & 0.18 & 0.22 & 0.25 \\
\hline
\end{tabular}




\section{Case 3: when constraints are in stochastic form}

The SP method is to deal with those decision-making optimization problems where the parameters of the problem assume continuous or discrete probability distributions. The stochastic optimization technique deals with the modelling and optimizing of decision-making problems where probability distributions control the data are known or may be estimated using unknown parameters. In many cases of TP, parameters have characterized as random variables instead of absolute values. In such TP, a stochastic optimization technique is used to solve the problem.

To discover the best fit distribution of constraints, we have tested five separate continuous statistical distributions. The best distribution for each constraint is determined based on the AIC and BIC. The distribution that results with the minimum value of AIC and BIC is concluded as the best fit distribution. Once the most suitable distribution has obtained, it is available to find the next suitable distributions. A difference factor $\left(\Delta=A I C-A I C_{\text {min }}\right)$ is calculated for every distribution tested for each constraint. A difference of 0 means best-fitted distribution, difference ranging between $0 . .2$ implies substantial fit, a range of $4 \ldots 7$ indicates lower support for the distribution and a difference above 10 shows no support at all.

In order, to obtain the lower and upper bound (pay off matrix) of each objective functions; we solved each objective separately under the same set of constraints by using optimization software LINGO 16.0. The values are given below:

Weibull:

$40388.12 \leq Z_{1} \leq 70855.48$,

$2295711 \leq Z_{2} \leq 3899169$;

Extreme value:

$40394.46 \leq Z_{1} \leq 69522.80$,

$2304326 \leq Z_{2} \leq 3844281$;

Cauchy:

$33989.08 \leq Z_{1} \leq 71310.91$,

$1907326 \leq Z_{2} \leq 3841019$;

Pareto:

$58142.86 \leq Z_{1} \leq 66646.61$,

$3455787 \leq Z_{2} \leq 3949635$;

Normal:

$40902.47 \leq Z_{1} \leq 56921.0$,

$2328205 \leq Z_{2} \leq 3179955$.

In this, the case we have considered the constraints (demand and supply) of the MOTP are not known or fixed. In such situations, the quantity of demand and supply is supposed to be random variables; this uncertainty expressed by probability distributions. We have considered five different distribution functions and also try to find out the best fit probability distribution. After following the stepwise procedure discussed in Section 5, we obtained the optimum solution. The discussed model is solved by using optimization software LINGO 16.0:
Weibull:

$Z_{1}=56498.78$,

Extreme value:

Cauchy:

$Z_{2}=3051332$;

$Z_{1}=55762.11$,

$Z_{1}=53887.73$,

$x_{11}=10$,

$Z_{2}=3131864$;

$Z_{2}=2809897$;

$x_{12}=0$,

$x_{13}=433$,

$x_{21}=0$,

$x_{22}=989$,

$x_{23}=0$,

$x_{31}=1018$,

$x_{32}=0$,

$x_{33}=411$;

Pareto:

$Z_{1}=58156.176$,

$x_{11}=16$,

$x_{12}=0$,

$x_{11}=1$,

$x_{12}=0$,

$x_{13}=470$,

$x_{21}=0$,

$x_{22}=979$,

$x_{23}=0$,

$x_{31}=969$,

$x_{32}=0$,

$x_{33}=412$;

$x_{13}=249$,

$x_{21}=0$,

$x_{22}=982$,

$x_{23}=0$,

$x_{31}=1080$,

$x_{32}=0$,

$x_{33}=320$;

Normal:

$Z_{1}=40909.95$,

$Z_{2}=3456918$;

$Z_{2}=2756953$;

$x_{11}=825$,

$x_{11}=0$,

$x_{12}=205$,

$x_{12}=0$,

$x_{13}=197$,

$x_{21}=0$,

$x_{22}=1012$,

$x_{23}=0$,

$x_{31}=0$,

$x_{32}=0$,

$x_{33}=1328$;

$x_{13}=877$,

$x_{21}=140$,

$x_{22}=282$,

$x_{23}=3$,

$x_{31}=731$,

$x_{32}=455$,

$x_{33}=0$.

Table 9 provides the availability of demand and supply. Values of parameters for all distributions with defined likelihood level and deterministic RHS of constraints are provided in Table 10. After using Equations (22) and (23) values of AIC and BIC are obtained and shown in Tables 11 and 12.

From Tables 11 and 12, the extreme value distribution has the minimum AIC and BIC for the value of $a_{1}$. Similarly, normal distribution for $a_{2}$, normal distribution for $a_{3}$, Weibull distribution for $b_{1}$, Weibull distribution for $b_{2}$, normal distribution for $b_{3}$. These are the best distributions for the specified constraints.

The best model and the next most suitable models are determined using AIC, BIC and Akaike weights, and these are depicted in Figure 2. In every graph, the best model curve is shown with black line and the original data is shown by a dark goldenrod coloured line. For $a_{1}$ the best model is extreme value distribution, and the next most suitable distributions are normal, logistic, Pareto and Weibull. Normal distribution fits $a_{2}, a_{3}$ and $b_{3}$ the best. For $b_{1}$ and $b_{2}$, Weibull distribution is the most proper distribution. The lowest AIC and BIC values determine the best-fitted distributions. Looking at the differences between AIC and minimum AIC for each constraint, the next most suitable models are obtained, and these can be viewed in Tables 13 and 14 .

After following the stepwise procedure discussed in Section 5, we obtained the compromise solution given in Table 15 and analysis of solution is presented in Figure 3. LINGO 16.0, optimization software has been used to get an optimum solution. 
Table 9. Availability of demand and supply

\begin{tabular}{|c|c|c|c|c|c||c|c|c|c|c|c|}
\hline$a_{1}$ & $a_{2}$ & $a_{3}$ & $b_{1}$ & $b_{2}$ & $b_{3}$ & $a_{1}$ & $a_{2}$ & $a_{3}$ & $b_{1}$ & $b_{2}$ & $b_{3}$ \\
\hline 833 & 1042 & 1540 & 956 & 1177 & 1269 & 968 & 1003 & 1688 & 728 & 1043 & 800 \\
\hline 1142 & 881 & 902 & 1050 & 1125 & 1332 & 1085 & 1020 & 1081 & 742 & 1374 & 986 \\
\hline 855 & 954 & 1339 & 1033 & 1146 & 1218 & 1090 & 992 & 1411 & 806 & 841 & 1205 \\
\hline 945 & 760 & 1159 & 912 & 951 & 1120 & 846 & 1066 & 1553 & 800 & 626 & 1289 \\
\hline 1001 & 783 & 1226 & 999 & 967 & 1068 & 983 & 790 & 1090 & 707 & 1215 & 1009 \\
\hline 1099 & 968 & 1634 & 1010 & 974 & 949 & 1068 & 785 & 1000 & 959 & 1133 & 805 \\
\hline 1309 & 1061 & 1681 & 801 & 784 & 952 & 996 & 854 & 1372 & 805 & 1293 & 1117 \\
\hline 1282 & 1060 & 1291 & 833 & 1208 & 1221 & 1218 & 856 & 919 & 1089 & 732 & 1212 \\
\hline 876 & 957 & 1015 & 891 & 1142 & 1358 & 1182 & 1064 & 933 & 1086 & 932 & 968 \\
\hline 1170 & 784 & 981 & 1044 & 873 & 1056 & 1394 & 866 & 1038 & 949 & 650 & 1392 \\
\hline 1125 & 1048 & 1070 & 1093 & 1084 & 997 & 830 & 824 & 955 & 786 & 1173 & 1104 \\
\hline 1075 & 1027 & 1438 & 939 & 600 & 1098 & 1151 & 983 & 1604 & 1043 & 1332 & 1273 \\
\hline 1216 & 846 & 982 & 757 & 913 & 1389 & 831 & 942 & 1249 & 725 & 1371 & 1302 \\
\hline 1055 & 841 & 1691 & 898 & 681 & 1140 & 1202 & 813 & 1332 & 892 & 661 & 1239 \\
\hline 858 & 1004 & 1582 & 1058 & 851 & 1015 & 1016 & 747 & 1686 & 856 & 901 & 1374 \\
\hline 904 & 863 & 1237 & 1056 & 1201 & 1300 & 1370 & 796 & 1219 & 830 & 1285 & 1018 \\
\hline 1065 & 928 & 1409 & 963 & 1040 & 962 & 1215 & 952 & 1677 & 871 & 943 & 838 \\
\hline 921 & 1030 & 1144 & 944 & 1167 & 1094 & 881 & 931 & 1127 & 795 & 853 & 801 \\
\hline 866 & 867 & 1324 & 832 & 870 & 884 & 1045 & 901 & 1203 & 1029 & 888 & 955 \\
\hline 1162 & 766 & 1527 & 1098 & 708 & 1379 & 1079 & 849 & 936 & 979 & 1357 & 1369 \\
\hline 967 & 711 & 1210 & 1059 & 1097 & 1040 & 1048 & 851 & 1628 & 811 & 768 & 1353 \\
\hline 863 & 815 & 1517 & 1064 & 630 & 1031 & 840 & 1012 & 1559 & 772 & 899 & 1077 \\
\hline 816 & 833 & 915 & 865 & 1136 & 1113 & 909 & 990 & 1405 & 1035 & 1356 & 1059 \\
\hline 1095 & 758 & 1323 & 957 & 1076 & 1049 & 1132 & 1000 & 1575 & 1080 & 1101 & 1373 \\
\hline 988 & 826 & 1297 & 749 & 643 & 1370 & 936 & 895 & 949 & 710 & 1364 & 995 \\
\hline
\end{tabular}

Table 10. Values of parameters with distributions

\begin{tabular}{|c|c|c|c|c|c|c|}
\hline $\begin{array}{l}\text { Probability } \\
\text { distribution }\end{array}$ & $\begin{array}{l}\text { Random } \\
\text { variables }\end{array}$ & Shape parameter & Scale parameter & $\begin{array}{l}\text { Location } \\
\text { parameter }\end{array}$ & $\begin{array}{c}\text { Specified probability } \\
\text { level }\end{array}$ & $\begin{array}{c}\text { Deterministic RHS } \\
\text { values }\end{array}$ \\
\hline \multirow{6}{*}{ Weibull } & $a_{1}$ & 7.23412 & 1102.19 & - & 0.88 & 1222.85 \\
\hline & $a_{2}$ & 10.1178 & 949.439 & - & 0.78 & 989.176 \\
\hline & $a_{3}$ & 5.80018 & 1397.58 & - & 0.68 & 1429.39 \\
\hline & $b_{1}$ & 8.77822 & 968.265 & - & 0.66 & 876.081 \\
\hline & $b_{2}$ & 5.0586 & 1093.40 & - & 0.87 & 740.507 \\
\hline & $b_{3}$ & 7.42876 & 1200.88 & - & 0.93 & 843.615 \\
\hline \multirow{6}{*}{ Extreme value } & $a_{1}$ & - & 125.997 & 964.161 & 0.88 & 1223.34 \\
\hline & $a_{2}$ & - & 90.4754 & 853.956 & 0.78 & 979.94 \\
\hline & $a_{3}$ & - & 224.832 & 1167.01 & 0.68 & 1381.23 \\
\hline & $b_{1}$ & - & 111.317 & 853.931 & 0.66 & 845.487 \\
\hline & $b_{2}$ & - & 212.721 & 888.078 & 0.87 & 736.402 \\
\hline & $b_{3}$ & - & 162.068 & 1039.69 & 0.93 & 881.18 \\
\hline \multirow{6}{*}{ Cauchy } & $a_{1}$ & - & 102.906 & 1031.12 & 0.88 & 1291.03 \\
\hline & $a_{2}$ & - & 77.3216 & 889.308 & 0.78 & 982.774 \\
\hline & $a_{3}$ & - & 187.184 & 1282.13 & 0.68 & 1400.92 \\
\hline & $b_{1}$ & - & 95.123 & 921.857 & 0.66 & 869.563 \\
\hline & $b_{2}$ & - & 165.134 & 1016.02 & 0.87 & 634.418 \\
\hline & $b_{3}$ & - & 117.888 & 1095.42 & 0.93 & 568.019 \\
\hline \multirow{6}{*}{ Pareto } & $a_{1}$ & 4.37666 & 816.00 & - & 0.88 & 1324.6 \\
\hline & $a_{2}$ & 4.27783 & 711.00 & - & 0.78 & 1012.95 \\
\hline & $a_{3}$ & 2.94186 & 902.00 & - & 0.68 & 1328.67 \\
\hline & $b_{1}$ & 4.01989 & 707.00 & - & 0.66 & 924.635 \\
\hline & $b_{2}$ & 2.05834 & 600.00 & - & 0.87 & 1616.67 \\
\hline & $b_{3}$ & 3.03056 & 800.00 & - & 0.93 & 1923.87 \\
\hline \multirow{6}{*}{ Normal } & $a_{1}$ & - & 149.632 & 1036.06 & 0.88 & 877.692 \\
\hline & $a_{2}$ & - & 100.921 & 903.9 & 0.78 & 833.477 \\
\hline & $a_{3}$ & - & 253.506 & 1292.46 & 0.68 & 1187.11 \\
\hline & $b_{1}$ & - & 121.627 & 914.92 & 0.66 & 870.442 \\
\hline & $b_{2}$ & - & 228.237 & 1002.7 & 0.87 & 736.495 \\
\hline & $b_{3}$ & - & 173.038 & 1126.34 & 0.93 & 879.568 \\
\hline
\end{tabular}



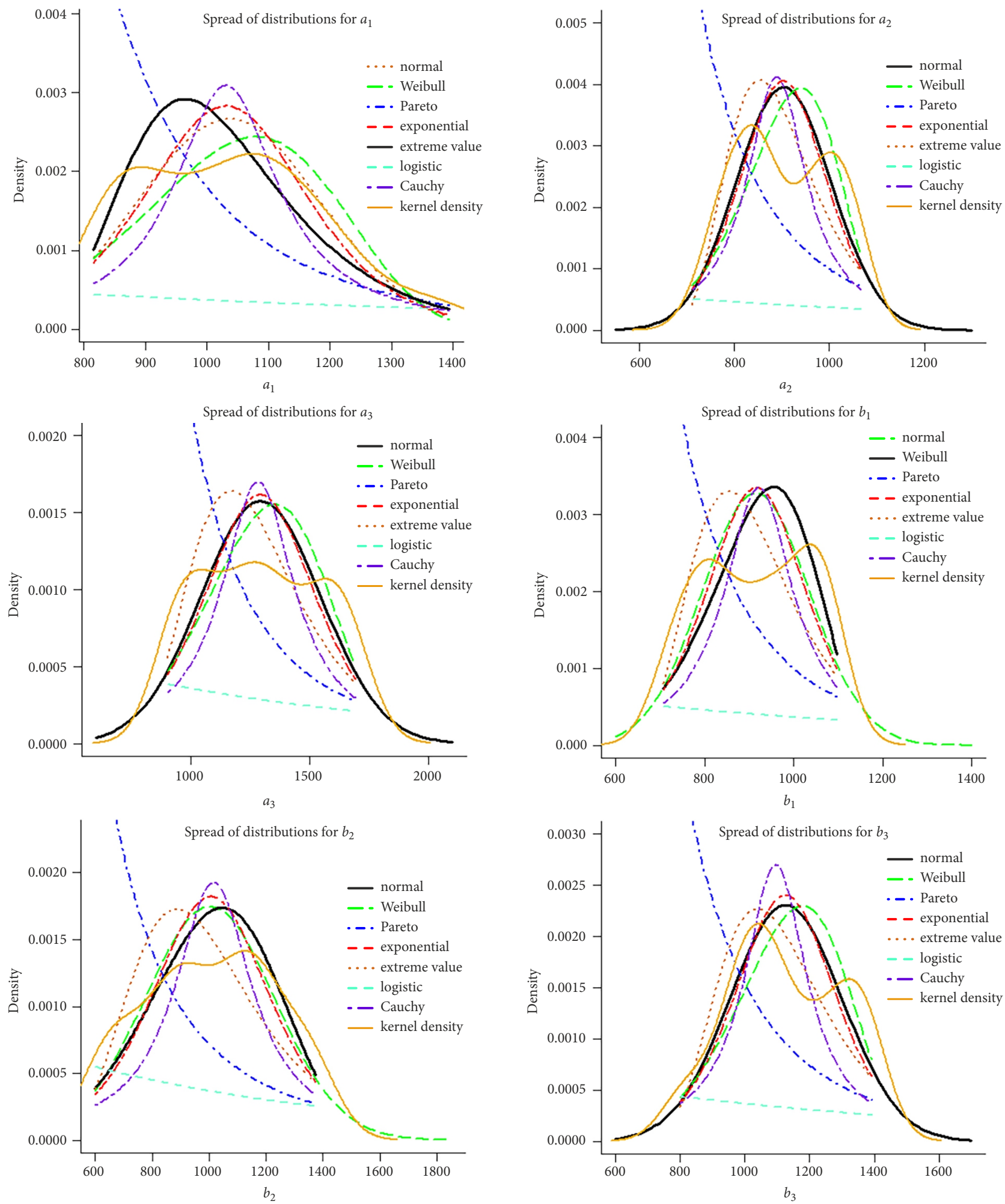

Figure 2. Spread of distributions for constraints

Table 11. AIC of the data set

\begin{tabular}{|l|c|c|c|c|c|c|}
\hline \multicolumn{1}{|c|}{ Distribution } & $a_{1}$ & $a_{2}$ & $a_{3}$ & $b_{1}$ & $b_{2}$ & $b_{3}$ \\
\hline Weibull & 651.8605 & 608.7026 & 699.6887 & 625.4354 & 687.9826 & 661.5466 \\
\hline Extreme value & 644.6901 & 609.7100 & 701.2756 & 630.0263 & 693.8819 & 666.1192 \\
\hline Cauchy & 671.0197 & 637.8488 & 728.8230 & 657.2916 & 716.0416 & 687.5926 \\
\hline Pareto & 649.6615 & 638.6991 & 710.5494 & 645.8538 & 720.0861 & 694.5837 \\
\hline Normal & 646.7118 & 607.3281 & 699.4324 & 625.9900 & 688.9322 & 661.2447 \\
\hline
\end{tabular}


Table 12. BIC of the data set

\begin{tabular}{|l|c|c|c|c|c|c|}
\hline \multicolumn{1}{|c|}{ Distribution } & $a_{1}$ & $a_{2}$ & $a_{3}$ & $b_{1}$ & $b_{2}$ & $b_{3}$ \\
\hline Weibull & 655.4292 & 612.5266 & 703.2565 & 629.2595 & 691.8066 & 665.3707 \\
\hline Extreme value & 648.5141 & 613.534 & 705.0997 & 633.8504 & 697.7060 & 669.9432 \\
\hline Cauchy & 674.8437 & 641.6729 & 732.6470 & 661.1157 & 719.8656 & 691.4166 \\
\hline Pareto & 653.4855 & 642.5231 & 714.3735 & 649.6779 & 723.9101 & 698.4078 \\
\hline Normal & 650.5358 & 611.1521 & 703.2565 & 629.8140 & 692.7562 & 665.0687 \\
\hline
\end{tabular}

Table 13. Differences $\Delta$ between AIC values

\begin{tabular}{|l|c|c|c|c|c|c|}
\hline \multicolumn{1}{|c|}{ Distribution } & $a_{1}$ & $a_{2}$ & $a_{3}$ & $b_{1}$ & $b_{2}$ & $b_{3}$ \\
\hline Weibull & 6.915 & 1.3745 & 0.0523 & 0 & 0 & 0.3019 \\
\hline Extreme value & 0 & 2.3819 & 1.8432 & 4.5909 & 5.8993 & 4.8745 \\
\hline Cauchy & 26.3296 & 30.5207 & 29.3906 & 31.8562 & 28.059 & 26.3479 \\
\hline Pareto & 4.9714 & 31.371 & 11.117 & 20.4184 & 32.1035 & 33.339 \\
\hline Normal & 2.0217 & 0 & 0 & 0.5546 & 0.9496 & 0 \\
\hline
\end{tabular}

Table 14. Akaike weights [\%]

\begin{tabular}{|l|c|c|c|c|c|c|}
\hline \multicolumn{1}{|c|}{ Distribution } & $a_{1}$ & $a_{2}$ & $a_{3}$ & $b_{1}$ & $b_{2}$ & $b_{3}$ \\
\hline Weibull & 41.16 & 26.51 & 39.48 & 52.11 & 56.76 & 41.16 \\
\hline Extreme value & 4.18 & 16.01 & 16.12 & 5.24 & 2.97 & 4.18 \\
\hline Cauchy & 9.09 & 1.24 & 1.68 & 6.35 & 4.58 & 9.09 \\
\hline Pareto & 2.76 & 8.12 & 0.15 & 0.0019 & 6.065 & 2.76 \\
\hline Normal & 47.87 & 52.70 & 40.53 & 39.49 & 35.30 & 47.87 \\
\hline
\end{tabular}

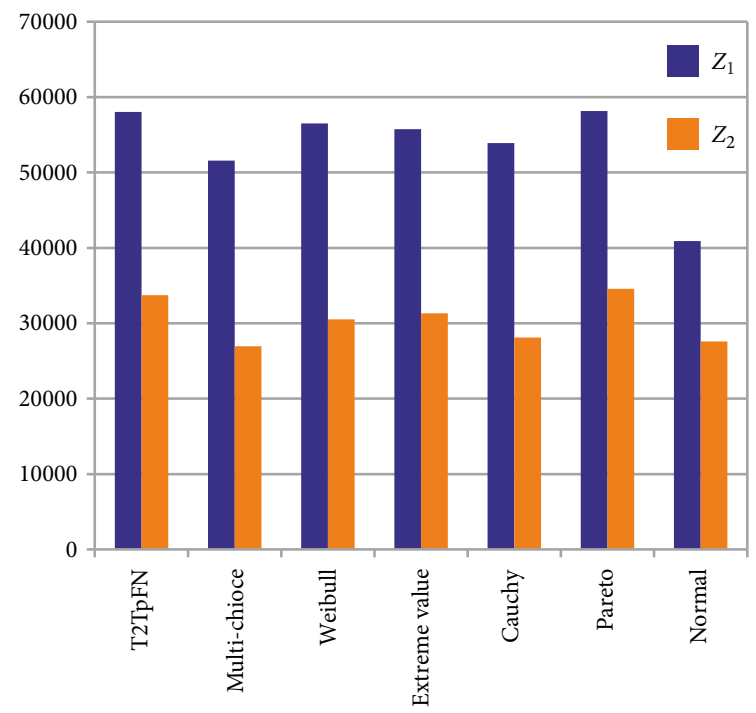

Figure 3. Analysis of solutions

\section{Discussion and analysis}

In this proposed work, we have considered a MOTP with conflicting objectives and mixed constraints. The objective functions of the formulated model are uncertain. T2TpFN used to expresses this uncertainty. We used a defuzzification technique to convert the T2TpFN into crisp value discussed by Biswas and De (2018). In this proposed study, we have also discussed three different cases.
"») when the constraints (demand and supply) of the model are T2TpFN;

"» when the constraints (demand and supply) of the model are multi-choice;

"») when the constraints (demand and supply) of the model are in stochastic form and dealt by five different probability distributions.

We have considered all three above situations and proposed models for MOTP. We have considered a suitable example for all three situations to show the applicability of the proposed work, in many real-life situations where the parameters of MOTP are precisely not known or uncertain. In those situations, uncertainty can be expressed by fuzziness, multi-choices and probability distribution, respectively.

Case 1. In this case, we have considered a situation where the input information about the parameters of the MOTP is not known or fixed. However, anyhow we can find some range about the parameters. Therefore, this uncertainty is presented by T2TpFN and transformed into a crisp value by a defuzzification technique discussed by Biswas and De (2018).

Case 2. In this case, we have considered a situation where the constraints (demand and supply) of the MOTP are in multi-choices. Therefore we used a transformation technique discussed by Roy and Maity (2017) to convert the multi-choices into a crisp value. 
Case 3. In this case, we have considered a situation where the constraints (demand and supply) of the MOTP are random variables. Five different types of probability distribution functions have been discussed. We have also used AIC and BIC technique to find the best probability distributions among them.

Therefore, discussing all three cases, we have followed the stepwise procedure of Section 6, to find the lower and upper bound and compromise solutions.

From Table 15, we can observe that the value of the first objective (profit maximization)is maximum when we consider the stochastic form with Pareto distribution. That means the model with the stochastic form with Pareto distribution gives the maximum profit as compared to the other models. From Table 15, we can also observe that the value of the second objective is minimum when the proposed model with multi-choice. That means the model with multi-choice gives the minimum cost as compared to the other models. Figure 3 depicted the behaviour of objective functions in different uncertain conditions and probability distribution.

Table 15. Analysis of compromise solutions

\begin{tabular}{|c|c|c|}
\hline $\begin{array}{l}\text { Uncertainty/ } \\
\text { distributions } \\
\text { in parameters }\end{array}$ & $\begin{array}{l}\text { Optimal objective } \\
\text { function values }\end{array}$ & $\begin{array}{c}\text { Optimal } \\
\text { transportation plan }\end{array}$ \\
\hline T2TpFN & $\begin{array}{l}Z_{1}=57564.71 \\
Z_{2}=3279763\end{array}$ & $\begin{array}{l}x_{11}=4, \\
x_{12}=73, \\
x_{13}=735, \\
x_{21}=0, \\
x_{22}=913, \\
x_{23}=0, \\
x_{31}=922, \\
x_{32}=0, \\
x_{33}=407\end{array}$ \\
\hline Multi-choice & $\begin{array}{l}Z_{1}=51555.71 \\
Z_{2}=2693496\end{array}$ & $\begin{array}{l}x_{11}=2, \\
x_{12}=0, \\
x_{13}=0, \\
x_{21}=0, \\
x_{22}=1100, \\
x_{23}=0, \\
x_{31}=713, \\
x_{32}=0, \\
x_{33}=800\end{array}$ \\
\hline Weibull & $\begin{array}{l}Z_{1}=56498.78 \\
Z_{2}=3051332\end{array}$ & $\begin{array}{l}x_{11}=10, \\
x_{12}=0, \\
x_{13}=433, \\
x_{21}=0, \\
x_{22}=989, \\
x_{23}=0, \\
x_{31}=1018, \\
x_{32}=0, \\
x_{33}=411\end{array}$ \\
\hline Extreme value & $\begin{array}{l}Z_{1}=55762.11 \\
Z_{2}=3131864\end{array}$ & $\begin{array}{l}x_{11}=16, \\
x_{12}=0, \\
x_{13}=470, \\
x_{21}=0, \\
x_{22}=979, \\
x_{23}=0, \\
x_{31}=969, \\
x_{32}=0, \\
x_{33}=412\end{array}$ \\
\hline
\end{tabular}

End of Table 15

\begin{tabular}{|c|c|c|}
\hline $\begin{array}{l}\text { Uncertainty/ } \\
\text { distributions } \\
\text { in parameters }\end{array}$ & $\begin{array}{l}\text { Optimal objective } \\
\text { function values }\end{array}$ & $\begin{array}{c}\text { Optimal } \\
\text { transportation plan }\end{array}$ \\
\hline Cauchy & $\begin{array}{l}Z_{1}=53887.73 \\
Z_{2}=2809897\end{array}$ & $\begin{array}{l}x_{11}=1, \\
x_{12}=0, \\
x_{13}=249, \\
x_{21}=0, \\
x_{22}=982, \\
x_{23}=0, \\
x_{31}=1080, \\
x_{32}=0, \\
x_{33}=320\end{array}$ \\
\hline Pareto & $\begin{array}{l}Z_{1}=58156.176 \\
Z_{2}=3456918\end{array}$ & $\begin{array}{l}x_{11}=825, \\
x_{12}=205, \\
x_{13}=197, \\
x_{21}=0, \\
x_{22}=1012, \\
x_{23}=0, \\
x_{31}=0, \\
x_{32}=0, \\
x_{33}=1328\end{array}$ \\
\hline Normal & $\begin{array}{l}Z_{1}=40909.95 \\
Z_{2}=2756953\end{array}$ & $\begin{array}{l}x_{11}=0, \\
x_{12}=0, \\
x_{13}=877, \\
x_{21}=140, \\
x_{22}=282, \\
x_{23}=3, \\
x_{31}=731, \\
x_{32}=455, \\
x_{33}=0\end{array}$ \\
\hline
\end{tabular}

\section{Managerial implications, key contributions, limitations, comparison}

\subsection{Managerial implications}

There are several managerial implications of the proposed work as given below:

"» the proposed work has a powerful practical application in industrials transportation problems. This study helps the mangers from industries to find optimum order quantity of shipment from source to destination at minimum TC;

"» the proposed model is designed under fuzziness, multi-choice and probabilistic situations, that can help the managers for making better decisions in these types of situations;

"» in the presented model, TC, profit, demand and supply are considered with vagueness rather than exact value, that types of scenario provide a flexible view to the transportation managers to make a right decision under vagueness;

"» the proposed work helps the managers to select the best transportation route to deliver the products from sources to destinations at minimum TC;

") every industries or organization have their primary objective to maximize the profit; this study helps them to make a profit at minimum TC;

"» our study helps managers to make better decisions for industries under an uncertain environment in transportation problems and also provide the ideas 
on how to perform under such types of unexpected scenarios;

"» this study gives an idea about how to tackle those situations, where some additional cost may be added to every product due to damage, late delivery, etc.;

"» the proposed work helps the managers to make a better decision of multi conflictive objectives of transportation problem under fuzzy environment.

"» moreover, the proposed work is simple, easy to use and also helps to find solutions to other multi-criteria management decision-making problems;

"» our study may be useful to researchers and organizations to build transportation evaluation systems in a fuzzy environment.

\subsection{Contributions}

Here is a list of the key contributions of this study.

"» a mathematical model of MOTP is designed with objectives of profit maximization and cost minimization;

"») the proposed model is considered in an uncertain environment, where all involved parameters are in an uncertain nature and presented by T2FNS;

"») constraints of the proposed model are discussed with two situations: (1) multi-choice, (2) probabilistic random variables, where random variable follows some different types of probability distributions;

"» AIC and BIC approach is used for the best fit probability distributions of MOTP;

"» a new function, rate of increment in TC and rate of decrement in profit on transporting of each product due to the damage, late deliveries, weather conditions, and any other issues are discussed in the proposed model;

"» FGP is used to solve the proposed MOTP;

"» the proposed work helps to understand the concept of T2FS in MOTP;

"» T2FS minimizes the more uncertainty than T1FS. Thus, the DMs can make better decisions with the help of the proposed study.

\subsection{Limitations}

There are few limitations related to our study, which is defined as follows:

"» in our proposed model, we mainly considered two factors; future studies can also use some other factors like time, sustainability, etc.;

"') computational work in the proposed study has been based on a hypothetical case study. It would be better to expand this work supported by real data;

"» FGP approach has been used in proposed work limited to only linear membership function, but there are some others membership functions available in the literature review, in future research some other memberships function can also be used in the proposed model;
"'» the presented model is based on vagueness, but in some other situations, DMs have to face when the model is not in the form of vagueness.

\subsection{Comparison with previous works}

After analyzing the above literature reviews of the transportation models, the objective with profit maximization is very rare. The researchers have done most of the past works on TP with one objective (TC). In our proposed work, we have considered both objectives. These objectives are opposite to each other. In our proposed study, we have considered all types of uncertainty and the concept of T2FS in both objectives and constraints, which is a new investigation in TP as per our best knowledge.

\section{Conclusion and future work}

In the supply chain, networking of transportation planning is a critical decision-making problem. The decisionmaking of transportation planning of raw material to industries, and finished goods to customers, vendors, warehouses, etc. are very challenging to the top stakeholders of the companies. Every supply chain's efficiency is directly correlated with the effective use of transport. For maximum profitability, each company makes use of different transport modes and routes. A logistics department uses transport to minimize the overall cost of the goods to be delivered, thus maintaining a fair degree of accessibility for customers.

The TP is a crucial problem of supply management and networking optimization and had been used in different areas of production distribution system, networks, rail, etc. The presented work enlightens various types of real-world challenging decision-making environments of transportation planning.

The paper has focused on the issues of various types of vagueness in the information. The vagueness has presented by fuzzy patterns (T2TpFN), multi-choices and stochastic. The vagueness of the information has been removed using the appropriate methods. The multi-choices of the information are dealt with using the binary conversion method, and fuzziness T2TpFN of the information is removed using the two-phases defuzzification procedure. The Stochastic information is dealt with the probability distributions, whereas the $\mathrm{c}$ method used to estimate parameters of the distribution.

Moreover, to best-fit distribution to a particular situation is determined by AIC and BIC approaches. Finally, the resultant crisp problem is a multi-objective optimization problem; for the final solution, we have used the FGP approach. An essential fact about the proposed work is that we have considered two different objectives, which are opposite to each other. The input parameters of the designed model are considered in an uncertain environment. Moreover, to present the uncertainty, we have considered three different scenarios T2TpFN, multi-choice and sto- 
chastic. The main contribution of the proposed work in the MOTP can be defined as follows:

"» the proposed model is considered in an uncertain environment, where all involved parameters of the MOTP are uncertain - this uncertainty expressed by fuzzy, multi-choice and stochastic;

"»» AIC and BIC approach is used for the best fit probability distributions of MOTP;

"y a new function, rate of increment in TC and rate of decrement in profit on transporting of each product due to the damage, late deliveries, weather conditions, and any other issues are discussed in the proposed model;

"» the proposed work helps to understand the concept of T2FS in MOTP;

"» T2FS minimizes the more uncertainty than T1FS. Thus, the decision-makers can make better decisions with the help of the proposed study.

TCs represent a large percentage of the supply chain's total expense, managers need a thorough analysis to choose a suitable mode of transportation to reduce costs and monitor the amount of shipment. Supply chain managers should take advantage of emerging technology to minimize TCs and boost their efficiency of networks allocation. Uncertainty of demand and supply for transportation must be taken into account while designing transport networks. If vagueness in transportation is avoided, it will increase expenses the transportation. The proposed approach is very easy and can be applied in many areas such as reliability, inventory management, queuing theory, etc. In future work, the discussed work can also be expressed as a bi-level optimization problem, the multi-level optimization problem for more complex TP case studies.

\section{References}

Acharya, S.; Biswal, M. P. 2016. Solving multi-choice multi-objective transportation problem, International Journal of Mathematics in Operational Research 8(4): 509-527.

Akaike, H. 1974. A new look at the statistical model identification, in E. Parzen, K. Tanabe, G. Kitagawa (Eds.). Selected Papers of Hirotugu Akaike, 215-222.

https://doi.org/10.1007/978-1-4612-1694-0_16

Aneja, Y. P.; Nair, K. P. K. 1979. Bicriteria transportation problem, Management Science 25(1): 73-78.

https://doi.org/10.1287/mnsc.25.1.73

Appa, G. M. 1973. The transportation problem and its variants, Journal of the Operational Research Society 24(1): 79-99. https://doi.org/10.1057/jors.1973.10

Arsham, H.; Kahn, A. B. 1989. A simplex-type algorithm for general transportation problems: an alternative to stepping-stone, Journal of the Operational Research Society 40(6): 581-590. https://doi.org/10.1057/jors.1989.95

Barik, S. K. 2015. Probabilistic fuzzy goal programming problems involving pareto distribution: some additive approaches, Fuzzy Information and Engineering 7(2): 227-244. https://doi.org/10.1016/j.fiae.2015.05.007

Barik, S. K.; Biswal, M. P.; Chakravarty, D. 2011. Stochastic programming problems involving Pareto distribution, Journal of Interdisciplinary Mathematics 14(1): 40-56.

https://doi.org/10.1080/09720502.2011.10700734
Biswal, M. P.; Acharya, S. 2009. Multi-choice multi-objective linear programming problem, Journal of Interdisciplinary Mathematics 12(5): 606-637.

https://doi.org/10.1080/09720502.2009.10700650

Biswal, M. P.; Acharya, S. 2011. Solving multi-choice linear programming problems by interpolating polynomials, Mathematical and Computer Modelling 54(5-6): 1405-1412.

https://doi.org/10.1016/j.mcm.2011.04.009

Biswal, M. P.; Samal, H. K. 2013. Stochastic transportation problem with Cauchy random variables and multi choice parameters, Journal of Physical Sciences 17: 117-130.

Biswas, A.; De, A. K. 2018. A unified method of defuzzification for type-2 fuzzy numbers with its application to multiobjective decision making, Granular Computing 3(4): 301-318. https://doi.org/10.1007/s41066-017-0068-z

Biswas, A.; Modak, N. 2011. A fuzzy goal programming method for solving chance constrained programming with fuzzy parameters, Communications in Computer and Information Science 140: 187-196.

https://doi.org/10.1007/978-3-642-19263-0_23

Biswas, A.; Modak, N. 2017. On solving multiobjective transportation problems with fuzzy random supply and demand using fuzzy goal programming, International Journal of Operations Research and Information Systems 8(3): 54-81. https://doi.org/10.4018/ijoris.2017070104

Biswas, P.; Pal, B. B. 2019. A fuzzy goal programming method to solve congestion management problem using genetic algorithm, Decision Making: Applications in Management and Engineering 2(2): 36-53.

Bit, A. K.; Biswal, M. P.; Alam, S. S. 1992. Fuzzy programming approach to multicriteria decision making transportation problem, Fuzzy Sets and Systems 50(2): 135-141. https://doi.org/10.1016/0165-0114(92)90212-m

Chakraborty, A.; Chakraborty, M. 2010. Cost-time minimization in a transportation problem with fuzzy parameters: a case study, Journal of Transportation Systems Engineering and Information Technology 10(6): 53-63.

https://doi.org/10.1016/S1570-6672(09)60071-4

Chang, C.-T. 2007. Multi-choice goal programming, Omega 35(4): 389-396. https://doi.org/10.1016/j.omega.2005.07.009

Chang, C.-T. 2008. Revised multi-choice goal programming, Applied Mathematical Modelling 32(12): 2587-2595.

https://doi.org/10.1016/j.apm.2007.09.008

Charnes, A.; Cooper, W. W. 1954. The stepping stone method of explaining linear programming calculations in transportation problems, Management Science 1(1): 49-69.

https://doi.org/10.1287/mnsc.1.1.49

Clímaco, J. N.; Antunes, C. H.; Alves, M. J. 1993. Interactive decision support for multiobjective transportation problems, European Journal of Operational Research 65(1): 58-67. https://doi.org/10.1016/0377-2217(93)90144-C

Current, J.; Marsh, M. 1993. Multiobjective transportation network design and routing problems: taxonomy and annotation, European Journal of Operational Research 65(1): 4-19. https://doi.org/10.1016/0377-2217(93)90140-I

Current, J. Min, H. 1986. Multiobjective design of transportation networks: taxonomy and annotation, European Journal of Operational Research 26(2): 187-201. https://doi.org/10.1016/0377-2217(86)90180-3

Dantzig, G. B. 1963. Linear Programming and Extensions. RAND Corporation. 641 p. https://doi.org/10.7249/R366

Diaz, J. A. 1979. Finding a complete description of all efficient solutions to a multiobjective transportation problem, Ekonomicko-matematický obzor 15(1): 62-73. 
Diaz, J. A. 1978. Solving multiobjective transportation problems, Ekonomicko-matematický obzor 14(3): 267-274.

Dinagar, D. S.; Palanivel, K. 2009. The transportation problem in fuzzy environment, International Journal of Algorithms, Computing and Mathematics 2(3): 65-71.

Dutta, D.; Murthy, A. S. 2010. Multi-choice goal programming approach for a fuzzy transportation problem, International Journal of Research and Reviews in Applied Sciences 2(2): 132-139.

Ebrahimnejad, A. 2014. A simplified new approach for solving fuzzy transportation problems with generalized trapezoidal fuzzy numbers, Applied Soft Computing 19: 171-176. https://doi.org/10.1016/j.asoc.2014.01.041

El-Wahed, W. F. A. 2001. A multi-objective transportation problem under fuzziness, Fuzzy Sets and Systems 117(1): 27-33. https://doi.org/10.1016/S0165-0114(98)00155-9

Fazlollahtabar, H. 2018. Operations and inspection cost minimization for a reverse supply chain, Operational Research in Engineering Sciences: Theory and Applications 1(1): 91-107.

Gupta, A.; Kumar, A. 2012. A new method for solving linear multi-objective transportation problems with fuzzy parameters, Applied Mathematical Modelling 36(4): 1421-1430. https://doi.org/10.1016/j.apm.2011.08.044

Isermann, H. 1979. The enumeration of all efficient solutions for a linear multiple-objective transportation problem, Naval Research Logistics Quarterly 26(1): 123-139. https://doi.org/10.1002/nav.3800260112

Kaliski, J. A.; Ye, Y. 1993. A short-cut potential reduction algorithm for linear programming, Management Science 39(6): 757-776. https://doi.org/10.1287/mnsc.39.6.757

Kamal, M.; Gupta, S.; Chatterjee, P.; Pamucar, D.; Stevic, Z. 2019. Bi-level multi-objective production planning problem with multi-choice parameters: a fuzzy goal programming algorithm, Algorithms 12(7): 143.

https://doi.org/10.3390/a12070143

Kamal, M.; Jalil, S. A.; Muneeb, S. M.; Ali, I. 2018. A distance based method for solving multi-objective optimization problems, Journal of Modern Applied Statistical Methods 17(1): 21. https://doi.org/10.22237/jmasm/1532525455

Lee, S. M.; Moore, L. J. 1973. Optimizing transportation problems with multiple objectives, AIIE Transactions 5(4): 333338. https://doi.org/10.1080/05695557308974920

Li, L.; Lai, K. K. 2000. A fuzzy approach to the multiobjective transportation problem, Computers \& Operations Research 27(1): 43-57. https://doi.org/10.1016/S0305-0548(99)00007-6

Liu, S.-T. 2016. Fractional transportation problem with fuzzy parameters, Soft Computing 20(9): 3629-3636. https://doi.org/10.1007/s00500-015-1722-5

Liu, S.-T. 2003. The total cost bounds of the transportation problem with varying demand and supply, Omega 31(4): 247-251. https://doi.org/10.1016/S0305-0483(03)00054-9

Lukovac, V.; Popović, M. 2018. Fuzzy Delphi approach to defining a cycle for assessing the performance of military drivers, Decision Making: Applications in Management and Engineering 1(1): 67-81.

Maity, S.; Roy, S. K. 2019. A new approach for solving type-2fuzzy transportation problem, International Journal of Mathematical, Engineering and Management Sciences 4(3): 683-696. https://doi.org/10.33889//IJMEMS.2019.4.3-054

Maity, G.; Roy, S. K. 2016. Solving a multi-objective transportation problem with nonlinear cost and multi-choice demand, International Journal of Management Science and Engineering Management 11(1): 62-70.

https://doi.org/10.1080/17509653.2014.988768
Maity, G.; Roy, S. K. 2014. Solving multi-choice multi-objective transportation problem: a utility function approach, Journal of Uncertainty Analysis and Applications 2(1): 11.

https://doi.org/10.1186/2195-5468-2-11

Maity, G.; Roy, S. K.; Verdegay, J. L. 2016. Multi-objective transportation problem with cost reliability under uncertain environment, International Journal of Computational Intelligence Systems 9(5): 839-849.

https://doi.org/10.1080/18756891.2016.1237184

Mahapatra, D. R.; Roy, S. K.; Biswal, M. P. 2013. Multi-choice stochastic transportation problem involving extreme value distribution, Applied Mathematical Modelling 37(4): 22302240. https://doi.org/10.1016/j.apm.2012.04.024

Pamucar, D.; Ćirović, G. 2018. Vehicle route selection with an adaptive neuro fuzzy inference system in uncertainty conditions, Decision Making: Applications in Management and Engineering 1(1): 13-37.

Rani, D.; Gulati, T. R. 2014. A new approach to solve unbalanced transportation problems in imprecise environment, Journal of Transportation Security 7(3): 277-287.

https://doi.org/10.1007/s12198-014-0143-5

Rani, D.; Gulati, T. R. 2017. Time optimization in totally uncertain transportation problems, International Journal of Fuzzy Systems 19(3): 739-750. https://doi.org/10.1007/s40815-016-0176-y

Ringuest, J. L.; Rinks, D. B. 1987. Interactive solutions for the linear multiobjective transportation problem, European Journal of Operational Research 32(1): 96-106. https://doi.org/10.1016/0377-2217(87)90274-8

Roy, S. K. 2014. Multi-choice stochastic transportation problem involving Weibull distribution, International Journal of Operational Research 21(1): 38-58. https://doi.org/10.1504/IJOR.2014.064021

Roy, S. K.; Mahapatra, D. R.; Biswal, M. P. 2012. Multi-choice stochastic transportation problem with exponential distribution, Journal of Uncertain Systems 6(3): 200-213.

Roy, S. K.; Maity, G. 2017. Minimizing cost and time through single objective function in multi-choice interval valued transportation problem, Journal of Intelligent \& Fuzzy Systems 32(3): 1697-1709. https://doi.org/10.3233/JIFS-151656

Roy, S. K.; Maity, G.; Weber, G.-W. 2017a. Multi-objective twostage grey transportation problem using utility function with goals, Central European Journal of Operations Research 25(2): 417-439. https://doi.org/10.1007/s10100-016-0464-5

Roy, S. K.; Maity, G.; Weber, G. W.; Gök, S. Z. A. 2017b. Conic scalarization approach to solve multi-choice multi-objective transportation problem with interval goal, Annals of Operations Research 253(1): 599-620. https://doi.org/10.1007/s10479-016-2283-4

Safi, M. R.; Ghasemi, S. M. 2017. Uncertainty in linear fractional transportation problem, International Journal of Nonlinear Analysis and Applications 8(1): 81-93.

Sahoo, N. P.; Biswal, M. P. 2005. Computation of some stochastic linear programming problems with Cauchy and extreme value distributions, International Journal of Computer Mathematics 82(6): 685-698. https://doi.org/10.1080/00207160412331336080

Si, A.; Das, S.; Kar, S. 2019. An approach to rank picture fuzzy numbers for decision making problems, Decision Making: Applications in Management and Engineering 2(2): 54-64.

Sinha, B.; Das, A.; Bera, U. K. 2016. Profit maximization solid transportation problem with trapezoidal interval type-2 fuzzy numbers, International Journal of Applied and Computational Mathematics 2(1): 41-56. https://doi.org/10.1007/s40819-015-0044-8 
Stojić, G.; Sremac, S.; Vasiljković, I. 2018. A fuzzy model for determining the justifiability of investing in a road freight vehicle fleet, Operational Research in Engineering Sciences: Theory and Applications 1(1): 62-75.

Stone, M. 1979. Comments on model selection criteria of Akaike and Schwarz, Journal of the Royal Statistical Society: Series B (Methodological) 41(2): 276-278.

https://doi.org/10.1111/j.2517-6161.1979.tb01084.x

Vilela, M.; Oluyemi, G.; Petrovski, A. 2019. A fuzzy inference system applied to value of information assessment for oil and gas industry, Decision Making: Applications in Management and Engineering 2(2): 1-18.

Xie, F.; Butt, M. M.; Li, Z.; Zhu, L. 2017. An upper bound on the minimal total cost of the transportation problem with varying demands and supplies, Omega 68: 105-118.

https://doi.org/10.1016/j.omega.2016.06.007

Zadeh, L. A. 1965. Fuzzy sets, Information and Control 8(3): 338-353. https://doi.org/10.1016/S0019-9958(65)90241-X

Zangiabadi, M.; Maleki, H. R. 2013. Fuzzy goal programming technique to solve multiobjective transportation problems with some non-linear membership functions, Iranian Journal of Fuzzy Systems 10(1): 61-74.

https://doi.org/10.22111/ijfs.2013.155 